\title{
ABNabstracts
}

\section{Proceedings of the Association of British Neurologists, University of Oxford, 3 April - 5 April 2002}

\begin{abstract}
ABN MEDAL AWARD 2002
Dr Pauline Monro

Pauline Monro was an outstanding medical student, gaining first class honours in her BSc at University College London, and then distinction in both medicine and pathology when she qualified from University College Hospital in 1958. She was also a competitive swimmer, winning a gold medal in the World University Games at Dortmund in 1953.

Like many others, she was inspired to go into neurology by JZ Young whom she had heard-while still at school-give the Reith lectures. Despite the challenges at a time when there was less than a handful of female consultant neurologists in the United Kingdom, and an almost fatal illness late in her training, she was appointed a consultant to Atkinson Morley's Hospital, London, in 1970-the first woman to be appointed to the consultant staff of the St George's Hospitals.

At $\mathrm{AMH}$ she threw her energies into creating one of the best neurosciences centres in the United Kingdom, encouraging multidisciplinary team meetings before they were fashionable, and developing an integrated neurology course for the medical students. Such was her charisma, that I can actually remember the teaching round when she explained to us the difference between upper and lower motor neuron lesions when I was a St George's medical student in 1967. She retired from the NHS in 1993 and later became the first woman to be President of the Section of Neurosciences at the Royal Society of Medicine.

As a result of going on the $A B N$ visit to Leningrad in 1988, Pauline became passionately involved with helping the neurological services in what had by then become St Petersburg, as well as in Russia generally. She became so fluent in Russian from a standing start that she can now lecture easily in the language, bought a flat in St Petersburg, and befriended young Russian neuroscientists. All this culminated in the most deserved award of an MBE in 2000. This is really her life work, more than being an NHS consultant, however successful-as she told me recently "driving across Europe to Russia in an ambulance with Zimmer frames is much more interesting than being an NHS neurologist".

Despite the loss of Michael, her beloved husband and partner, in 2000, Pauline continues her work in Russia with as much energy as ever. I can't believe she will ever stop shuttling backwards and forwards to Russia with yet another small band of British physiotherapists or nurses going one way and their Russian counterparts coming the other. When Pauline first went to Russia, neurological care there had been isolated from the West for 70 years. Now, encouraged and helped by Pauline, the first multidisciplinary stroke team in Pavlov's Medical University Hospital is accepted by the local Committee of Health as a model centre, one of many examples of good practice which are springing up across the city. In St Petersburg she is known to everyone as Paulina, and so it is with great pleasure that I invite you-Paulina-to give your lecture entitled "Russian and British neurology: contacts and contrasts".

CHARLES WARLOW
\end{abstract}

\section{NEUROFERRITINOPATHY}

Chinnery PF, Curtis AR, Fey C, Jackson M, Bates D, Morris CM, Bindoff LA, Coulthard A, Ince PG, Curtis A, Burn J The University of Newcastle upon Tyne, UK

We have identified an adenine insertion at position 460-461 in the ferritin light polypeptide gene (FTL) in affected members of a large Cumbrian family with a late-onset movement disorder. The clinical features of this disease are highly variable. Some individuals presented with a focal dystonia in childhood which rapidly became generalised; others developed chorea in adult life; and others were diagnosed as having a late onset akinetic-rigid syndrome similar to idiopathic Parkinson's disease. Brain imaging was strikingly abnormal in some cases with cystic degeneration of the caudate and lentiform nucleii. Postmortem examination revealed a widespread abnormality of iron metabolism, particularly affecting the globus pallidus, which contained spherical inclusions of iron and ferritin. Following the identification of the first family, the same mutation has been identified in other patients throughout Britain. In addition to possible links to the United States, one member of the founder family was Fletcher Christian, who achieved fame as the leader of the mutiny on the Bounty. This raises the possibility that other cases of this disease may live in the South Pacific region. This family provides a direct link between a disorder of brain iron metabolism and a neurodegenerative movement disorder, and raises the possibility of treating the disease by modifying brain iron stores.

\section{THE GENETIC BASIS AND PHENOTYPIC VARIABILITY IN EARLY ONSET PARKINSONISM}

Khan N, Valente EM, Brooks DJ, Piccini P, Bhatia KP, Quinn NP, Wood NW Institute of Neurology, London, UK

Introduction: Three loci have been mapped in autosomal recessive juvenile parkinsonsim: PARK2 (parkin) on chromosme 6 and PARK6 and PARK7 both on chromsome 1. Mutations in PARK2, parkin, are a common cause of young onset parkinsonism. PARK6 has been linked to early onset parkinsonism to chromosome 1 in a Italian kindred. A further eight unrelated families from different parts of Europe have been shown to be linked to the same region suggesting that it may also account for a share of autosomal recessive parkinsonism.

Here we summarise the genetics, clinical phenotype and ${ }^{18} \mathrm{~F}$-dopa PET studies in both parkin disease and PARK6-linked parkinsonsim.

Methods: PARK2: we have identifed 24 patients with parkin mutations using sequencing and semi-quantitative PCR techniques. We have carried out a detailed clinical evaluation using a standardised clinical proforma. A cohort of these patients have undergone ${ }^{18} \mathrm{~F}$-dopa PET.

PARK6: a clinical evaluation of the Marsala family in which parkinsonism was first linked to chromosome 1 and other unrelated families linked to PARK6 was performed. Four patients with PARK6-linked parkinsonsim and three carriers of a single mutant PARK6 underwent ${ }^{18} \mathrm{~F}$ dopa PET.

Results: Parkin disease and PARK6-linked parkinsonsim are distinct genetic entities with an overlap in the clinical phenotype. We report three new phenotypes at presentation in parkin disease: exercise-induced dystonia, focal dystonia and autonomic neuropathy with peripheral neuropathy. Both PARK2 and PARK6-linked parkinsonism have nigrostraiatal F-dopa uptake in both caudate and putamen which is unlike that of IPD.

Conclusion: PARK6-linked parkinsonsim has a phenotype that shares features of both IPD and parkin disease ${ }^{18} \mathrm{~F}$-dopa PET however suggests that it is an atypical form of parkinsonism. Preliminary work suggests that PARK6 contributes to a share of young onset parkinsonism. Parkinsonism due to mutation in PARK2 is an important cause of young onset parkinsonism that should be considered in the diagnostic work up. 


\section{RE-EMERGENCE OF ENCEPHALITIS LETHARGICA: NEW EVIDENCE OF AN AUTOIMMUNE CNS DISEASE}

Dale RC, Church AN, Surtees RAH, Lees AJ, Neville BGR, Giovanni G Institute of Neurology; Institute of Child Health, London, UK

Background: Encephalitis lethargica (EL) is an acute encephalitic illness with somnolent-ophthalmoplegic, hyperkinetic and Parkinsonian phenotypes. Pathology reveals inflammatory disease of the basal ganglia and midbrain. The presence of CSF oligoclonal bands, and improvement after steroid treatment have suggested that EL may be immune-mediated.

Methods: We report on five children and one adult with EL. The serum and CSF was examined for the presence of anti-neuronal antibodies using western immunoblotting and immunofluorescence. 200 children and adult controls were used for comparison.

Results: All six patients had evidence of autoantibodies reactive against basal ganglia neurones in both serum and CSF. The basal ganglia antigens involved in antibody reactivity were of molecular weight 40,45 and $60 \mathrm{kDa}$. Positive binding was seen in $3 \%$ of paediatric and adult controls $(n=200)$. Three patients with the parkinsonian phenotype had a similar binding pattern to midbrain (substantia nigra) neurons. There was no binding to neural antigens from other brain regions. Four patients had evidence of a preceding streptococcal infection.

Conclusion: We propose that $\mathrm{EL}$ is a postinfectious autoimmune CNS disorder with auto-antibody targeting of the basal ganglia and substantia nigra neurons. EL is still endemic in the population with the potential to reappear in epidemic forms.

\section{NATURAL HISTORY OF MULTIPLE SCLEROSIS: IMPLICATIONS FOR INTERPRETATION OF RELAPSE SUPPRESSION}

Ebers GC University of Oxford, UK

Background: It is still widely believed that the progression of disability in multiple sclerosis results from accumulative effect of focal exacerbations. Although this is most clearly true for patients with the Devic's phenotype, it has not been formally shown in the great majority of MS patients who begin with exacerbations and subsequently develop secondary progressive disease (SPMS). It is clear that the number of exacerbations in the first 2 years has an important association with long term outcome. We examined here the association between relapses and the onset of progression, and in addition time to long term disability using DSS 6, 8, and 10 .

Methods: The study was population based and derived from a cohort of 1042 patients from the London, Ontario MS clinic. The follow up entails some 25000 patient years of observation. The patients were seen approximately yearly over their period of observation. The DSS scale (Kurtske) was used for measurement of disability.

Results: Although an association was found between early exacerbations and long term disability, this relationship did not appear to be causal. The total number of relapses through the course of the disease bore no relationship to long term outcome. The accumulation of disability appears to be secondary to a relapse-independent process.

Discussion: These findings imply that suppression of relapses will have little effect on long term disability and are consistent with an expectation of no effect. Modelling of outcome based on clinical features has identified characteristics which correlate with disability well in excess of $R=0.9$. These findings far surpass correlations with any surrogate markers. Since long term disability is primarily determined by the progressive phase of the illness, outcome measures need to be reconsidered and we propose some which seem to have more rationale than those presently in use.

\section{THE PREDICTIVE VALUE OF BRAIN LESION LOAD IN DETERMINING BRAIN ATROPHY IN MULTIPLE SCLEROSIS: 14 YEAR FOLLOW UP DATA FOLLOWING FIRST PRESENTATION WITH A CLINICALLY ISOLATED SYNDROME}

Chard DT, Brex PA, Ciccarelli O, Griffin CMB, Dalton C, Thompson AV, Miller DH Institute of Neurology, London, UK

Background: Studies suggest that brain lesion load and atrophy may be related in multiple sclerosis (MS), but the time course of this relationship is unclear. This study investigates the relationship between brain atrophy and lesion load in subjects followed up for 14 years after a clinically isolated syndrome (CIS).

Methods: Twenty nine control subjects and 31 subjects followed up after developing a CIS were studied. At 14 year follow up, four subjects remained diagnosed with a CIS, four had clinically probable and 23 had clinically definite MS. In patients, MRI determined T2 lesion load was assessed at baseline, 5, 10, and 14 years, along with brain volume as a fraction of total intracranial volume (brain parenchymal fraction [BPF]) at 14 years. Differences in MS BPF compared to controls were estimated.

Results: The Spearman correlation of change in T2 lesion load from baseline to 5 years with 14 year BPF reduction $\left(r_{s}=20.619\right.$ $p<0.001$ ) was stronger than for subsequent change in lesion load.

Discussion: Change in lesion load in the first 5 years after clinical onset is a stronger predictor of subsequent atrophy than later changes. This suggests that early inflammation influences later neurodegeneration in MS

\section{$06 \beta$-INTERFERON TREATMENT DOES NOT IMMEDIATELY SLOW THE PROGRESSION OF AXONAL INJURY IN MULTIPLE SCLEROSIS}

Parry A, Corkill R, Blamire A, Palace J, Narayanan S, Arnold D, Styles P, Matthews PM John Radcliffe Hospital, Oxford, UK; Raddliffe Infirmary, Oxford; McGill University; Montreal Neurological Hospital and Institute, Quebec

Progression of disability in multiple sclerosis (MS) appears related to axonal damage in white matter. Histopathological studies have linked inflammatory activity directly to axonal injury and transection. $\beta$-Interferon (BIFN) substantially reduces new inflammatory activity in MS

To test whether BIFN treatment can arrest the progression of axonal injury in patients with MS, 11 patients with active relapsing remitting MS were treated with BIFN and serial MRI and magnetic resonance spectroscopic measurements of $\mathrm{N}$-acetylaspartate (NAA) - a measure of axonal injury-were performed before and during treatment.

As expected relapse rate reduced ( $p=0.007$ ), measures of cerebral oedema (white matter T2 relaxation time) improved $(p=0.04)$ and lesion volume stabilised. In contrast, white matter NAA/creatine continued to decline $(p=0.02)$. Interestingly, the magnitude of the NAA/ creatine decrease was correlated with the frequency of relapses over the two years prior to treatment $(r=20.76, p=0.006)$.

Thus either both processes are occurring independently, or the consequences of reduced inflammation on axonal injury are substantially delayed. This important distinction requires further study.

\section{VALIDATION OF THE MCDONALD CRITERIA IN PATIENTS WITH CLINICALLY ISOLATED SYNDROMES}

Dalton CM, Brex PA, Miszkiel KA, Plant GT, Hickman SJ, MacManus DG, Thompson AJ, Miller DH. Institute of Neurology; Lysholm Department of Neuroradiology; Moorfields Eye Hospital, London, UK

Background: Traditionally, multiple sclerosis (MS) has been diagnosed on the basis of clinical evidence of dissemination in time and space. Recently the international panel on MS diagnosis published the McDonald criteria which have integrated MRI and included patients with clinically isolated syndromes (CIS).

Methods: Patients with CIS were imaged at baseline, 3 months, 1 year and 3 years to determine the frequency with which patients with CIS fufilled the traditional Poser criteria for clinically definite MS and the new McDonald criteria for MS. The reliability of the McDonald criteria was assessed using CDMS as the gold standard

Results: At 3 months 20/95 (21\%) had MS using the McDonald criteria whereas only $7 / 95(7 \%)$ had developed CDMS. After 3 years the corresponding figures were, $29 / 50(58 \%)$ and $19 / 50(38 \%)$. Development of MS using the McDonald criteria at 3 months had high specificity $(93 \%)$, positive predictive value $(85 \%)$, negative predictive value $(82 \%)$ and accuracy $(83 \%)$ but lower sensitivity $(65 \%)$ for CDMS at 3 years.

Discussion: Specificity is more important than sensitivity when diagnosing lifelong conditions such as MS. The specificity and positive predictive value of the McDonald criteria at 3 months for CDMS at 3 years was high, therefore the new criteria are valid. 


\section{ISOLATED ASYMMETRIC DOMINANTLY POSTURAL TEMOR WITH OR WITHOUT RESTING TREMOR: A VARIANT PRESENTATION OF LONG LATENCY TREMULOUS PARKINSON'S DISEASE? A CLINICAL FOLLOW UP}

Pal S, Peng R, Brooks DJ, Rao CS, Ray Chaudhuri K Kings College Hospital, London, UK; First University Hospital, Chengdu, China; Hammersmith Hospital, London

Background: Considerable diagnostic uncertainty exists in patients presenting with isolated asymmetric postural tremor (PT), with or without resting tremor (RT) in the absence of significant bradykinesia, or rigidity. Whether such patients demonstrate an "atypical" pattern of essential tremor (ET) or ultimately progress to develop Parkinson's disease (PD) and thus are misdiagnosed as ET, is unclear.

Methods: Twelve patients (seven male, mean age 55.7 (range 45-68)) initially presenting with isolated asymmetric PT $\pm R T$ and erroneously diagnosed as ET by neurlogists/geriatricians at presentation but developing signs of PD during clinical follow up or "re-referral" were identified from our clinical database.

Results: Mean duration of PT prior to diagnosis of PD was 12.8 years ranging up to 40 years (range 5-40). Six patients had a family history of ET and three a family history of PD. Nine cases demonstrated both postural and resting tremor, the remainder having asymmetric PT without bradykinesia at presentation. Alcohol or propanolol sensitivity was recorded in four patients with PT and RT. Dopaminergic therapy was of symptomatic benefit in all treated cases $(n=10)$.

Discussion: Tremulous PD should be considered in prognostic counselling of patients presenting with asymmetric isolated PT \pm RT even if other diagnostic criteria for PD are not satisfied.

\section{ANTIPARKINSONIAN ACTIONS OF THE SELECTIVE DOPAMINE D3 RECEPTOR ANTAGONIST S33084}

Silverdale MA, Millan M, Newman-Tancredi A, Crossman AR, Brotchie JM Manchester Movement Disorders Laboratory, UK

The significance of dopamine D3 receptors in the control of movement remains unclear. It has been suggested that D3 agonist properties of widely used D2/D3 agonists such as ropinirole (hD3, Ki=56 nM and $\mathrm{hD2}, \mathrm{Ki}=154 \mathrm{nM}$ ) and pramipexole (hD3, $\mathrm{Ki}=9 \mathrm{nM}$ and $\mathrm{hD} 2$, $\mathrm{Ki}=182 \mathrm{nM}$ ) account for their control of Parkinson's disease. The lack of highly selective dopamine D3 receptor antagonists has hampered study of their role. S33084 (hD3, $\mathrm{Ki}=0.4 \mathrm{nM}$ and $\mathrm{hD} 2, \mathrm{Ki}=57 \mathrm{nM})$ displays >100-fold selectivity for hD3 versus D2 receptors. These drugs were administered to 1-Methyl-4-phenyl-1, 2, 3, 6-tetrahydropyridine (MPTP) lesioned marmosets previously primed with levodopa to elicit dyskinesia. S33084 potentiated antiparkisonian actions of levodopa $(p<0.05)$ and ropinirole $(p<0.01)$. There was no significant effect on dyskinesia. The action of ropinirole was reduced by L741, 626 ( $p<0.05)$. Alone, S33084 exerted a modest but significant antiparkinsonian effect $(p<0.05)$. Employing selective D3 versus D2 receptor antagonists, we provide evidence that stimulation of D3 receptors compromises motor function. We conclude that clinically available D2/3 agonists exert antiparkinsonian actions via D2 rather than D3 receptors. Indeed, stimulation of D3 receptors may be detrimental to antiparkinsonian efficacy.

\section{CLINICAL FEATURES OF THE GESTE ANTAGONISTE IN CERVICAL DYSTONIA}

Filipovic SR, Jahanshahi M, Viswanathan R, Heywood P, Bhatia KP, Rogers D Institute of Neurology, London; Burden Neurological Institute and Frenchay Hospital, Bristol, UK

A unique feature of dystonia is that patients are often able to reduce/ eliminate the abnormal posture by using certain manoeuvres that involve tactile or proprioceptive stimulation. Although widely recognised, these manoeuvres or "gestes antagonistes" (GA), have not been systematically analysed. To assess this phenomenon in more detail, we developed and administered a questionnaire to 100 successive patients with idiopathic cervical dystonia (CD) attending two botulinum toxin clinics (London and Bristol). Almost 2/3 patients $(64 \%)$ reported a GA. There were no differences between patients with and without a GA in terms of age, sex, duration of disease, clinical presentation, and age at onset. Among those with a GA, 58\% discovered their GA within the year of $C D$ onset, and in the majority $(91 \%)$ the GA was still effective. The GA target spots were mostly located on the cheek $(53 \%)$ and chin $(43 \%)$, back of the neck $(45 \%)$ and head $(35 \%)$. The index $(60 \%)$ and middle $(48 \%)$ fingers and the palm $(40 \%)$ were the most used for eliciting the GA. Touching the GA target spot with an object was effective for $27 \%$, while other people were able to elicit the effect by touching the GA target in $27 \%$ of patients. The majority $(84 \%)$ reported that the GA improves their $C D$ by at least $50 \%$, while almost one third $(30 \%)$ felt that the GA improves their CD "greatly" (ie $>75 \%$ ) or "completely". The GA effect was judged to be slightly better for improving head/neck/ shoulder position than for changing neck and shoulder movements and pain.

The almost ubiquitous presence and remarkable efficacy of the GA in $C D$ warrants further investigation of its physiology which may provide clues for understanding the neurobiology of dystonia and open new avenues for its treatment.

\section{IS DYSTONIA A DISORDER OF MUSCLE?}

Frima N, Grunewald RA University of Sheffield, UK

Background: The vibration induced illusion of movement (VIIM) is subserved by muscle spindle la afferents, and is subnormal in dystonic subjects. We examined the effect of muscle fatigue on VIIM and its perception in asymptomatic first degree relatives.

Methods: Nineteen patients with torticollis (mean age 55.37) were compared to 18 healthy control subjects (mean age 58.9). Nine parents, 20 siblings and 11 children were compared to 13 dystonic probands (mean age 55) and healthy control subjects. $80 \mathrm{~Hz}$ vibration was applied to the biceps brachii tendon with the arm fixed in a splint. Relaxation of the arm was verified by surface EMG. Blindfolded subjects were asked to copy the perceived movement of the vibrated arm with their other arm. Extension of the tracking arm was measured over 45 seconds. The task was repeated following fatigue of the vibrated arm.

Results: Perception of VIIM was less in dystonic patients $\left(19.1^{\circ}+/ 21.86\right.$; mean change of angle $\pm 2 S E$ ) than controls( $28.81^{\circ}+/ 21.74$; mean $\left.\pm 2 \mathrm{SE}\right), \quad(p<0.01$, unpaired $t$ test $)$, but increased in dystonic subjects when the vibrated arm was fatigued $\left(24.68^{\circ} \pm 22.5\right.$; mean $+/ 2 \mathrm{SE}, \mathrm{p}=0.01$, paired $t$ test compared with prefatigued values). (2/13) $85 \%$ of index patients, $(19 / 37) 51 \%$ of first degree relatives, and $(5 / 18) 28 \%$ of healthy controls had abnormal VIIM.

Discussion: These results imply that abnormal muscle spindle function in dystonia is genetically determined, and that muscle fatigue has a role in the genesis of dystonia in predisposed individuals.

\section{ANTIEPILEPTIC DRUGS IN PREGNANCY: UPDATE OF THE UK EPILEPSY AND PREGNANCY REGISTER}

Craig J, Russell A, Morrison P, Guthrie E, Parsons L, Waddell R, Morrow J Royal Group of Hospitals, Belfast, Northern Ireland, UK

Objective: To determine the relative risks for major congenital malformations (MCM) of in utero exposure to antiepileptic drugs (AEDs)

Design-Prospective, observational, registration and follow up study.

Subjects: -Women with epilepsy who become pregnant, whether or not they are taking an AED, either singly or in combination and who are referred before outcome of the pregnancy is known.

Outcome measure: MCM for each AED regime for exposed pregnancies.

Results: Full outcome data are available on 1714 pregnancies from 1996 through November 2001, with 595 outcomes awaited Monotherapy exposures account for $72 \%$ (MCM rate $4.4 \%[95 \% \mathrm{Cl}$ $3.4-5.8 \%]$ ), polytherapy exposures for $23 \%$ (MCM rate $5.3 \%[95 \%$ Cl $3.4-8.2 \%]$ ), and no AED exposures for 5\% (MCM rate 1.1\% [95\% $\mathrm{Cl} 0.1-6.2 \%]$ ) of cases. Although the MCM rate for carbamazepine (3.1\% [95\% Cl 1.9-5.1\%] and lamotrigine $(2.8 \%[95 \% \mathrm{Cl} 1.3-6.0 \%)$ are less than that recorded for sodium valproate $17.3 \%$ [95\% Cl 5.0$10.4 \%]$ ) the differences are not statistically significant. 
Conclusions: The UK Epilepsy and Pregnancy Register is proving an effective method of collecting outcome data on large numbers of pregnancies occurring in women with epilepsy. The results suggest that there are possible differences in MCM rate between AEDs, but further cases are required to establish the degree of these differences and the influence of other confounding variables.

\section{NURSE LED EPILEPSY CLINICS: A TELEMEDICINE APPROACH}

Bingham E, Patterson V. Royal Victoria Hospital, Belfast, Northern Ireland, UK

Patients with epilepsy consistently express a desire to see nurse specialists as well as a neurologist. We have developed nurse led epilepsy clinics at two rural hospitals, supported by telephone and videolink to a distant neurologist and here report on the feasibility, satisfaction, and cost of this method. An experienced epilepsy nurse (EB) assessed review patients with epilepsy at the clinics of a consultant neurologist (VP) at district general hospitals 75 and 85 miles respectively from the Neurological Centre. If further advice was needed, she telephoned the distant neurologist. If necessary the neurologist arranged a real time videolink with the nurse and patient. Satisfaction was assessed by a simple questionnaire using a three point Likert scale. The time spent by the patient with the nurse and the neurologist was documented and costed. One hundred and fifty five patients were seen over an 11 month period. Fifty five $(35 \%)$ were managed solely by the nurse, $55(35 \%)$ needed a telephone call, and $45(29 \%)$ required a video link. Seventy six per cent of all patients preferred the telemedicine. Neurologist time was reduced by $80 \%$. Overall cost increased from $£ 12.87$ to $£ 17.59$ per patient. Nurse led epilepsy clinics supported by a distant consultant neurologist using telemedicine, are feasible, more acceptable, but more expensive than conventional care.

\section{QUANTITATIVE VALIDATION OF MR INTENSITY ABNORMALITIES IN VARIANT CJD}

Colchester ACF, Hoijat SA, Will RG, Collie DA University of Kent, UK, and Western General Hospital, Edinburgh, Scotland, UK

High signal in the posterior thalamus on MRI scanning has emerged as an important diagnostic sign in variant Creutzfeldt-Jakob disease. We carried out quantitative analysis of 39 dual echo (PD and T2) MRI scans from a variety of scanners. 11 were from proven $\mathrm{VCJD}$ patients and 28 were from controls $(12$ sCJD and 16 non-CJD dementia cases). Absolute intensities vary greatly between scans, and the usefulness of different strategies for estimating relative intensities was examined.

Calculating the posterior thalamus to putamen intensity ratio on T2 scans, and using a threshold of 1.0 , was $100 \%$ sensitive for vCJD against controls but the specificity was only $20 \%$; while using the caudate instead of the putamen as a reference structure gave a good balance of sensitivity $(82 \%)$ and specificity $(93 \%)$. On PD scans the putamen was the better reference structure, giving $91 \%$ and $86 \%$ respectively. The best diagnostic discrimination overall was achieved with the posterior thalamus to frontal white matter ratio on $\mathrm{T} 2$ scans by using a threshold of 1.51, giving 100\% sensitivity and $89 \%$ specificity.

These quantitative results, on scans acquired at many different centres, have provided objective validation of published visual judgements and have confirmed that use of non-unity thresholds can improve discrimination further.

\section{MAPPING THE EVOLUTION OF REGIONAL ATROPHY OF REGIONAL ATROPHY IN ALZHEIMER'S DISEASE USING FLUID REGISTERED MRI}

Schott JM, Scahill RI, Stevens JM, Rossor MN, Fox NC Dementia Research Group, National Hospital for Neurology and Neurosurgery, London, UK

Objective: To assess altering patterns of significant regional atrophy during the progression of Alzheimer's disease (AD)

Methods: Patients with presymptomatic $A D(n=4)$, mild $A D(n=10)$ and moderate $A D(n=12)$ were compared with age and sex matched controls. All subjects had two serial MRI scans. Non-linear (fluid) registration was performed on the scan pairs, and statistical parametric mapping was used to demonstrate regions showing consistent significant $(p<0.001)$ expansion or contraction between each group and controls.
Results: We demonstrated increasing global atrophy with advancing disease. In presymptomatic individuals rates of atrophy were significantly increased in both hippocampi and the precuneus. In mildly and moderately affected patients the most significantly increased rates of atrophy were seen in the inferior and lateral temporal lobes, precuneus, and cingulate.

Conclusions: We demonstrated changes in the localisation of the most active areas of volume loss as AD progresses. Whilst measures of hippocampal change may be most useful at the earliest stages of the disease, the precuneus and posterior cingulate, which show significant, consistent and increasing rates of atrophy with increasing severity may prove to be useful indices of progression throughout the course of the disease.

\section{EARLY EXPERIENCE WITH MITOXANTRONE IN AGGRESSIVE RELAPSING-REMITTING MULTIPLE SCLEROSIS}

Ezhil V, Boggild M The Walton Centre for Neurology and Neurosurgery, Liverpool, UK

Background: Data from three randomised controlled trials supports the use of mitoxantrone, a cytotoxic agent, in active relapsing and secondary progressive multiple sclerosis (MS). The drug has recently been licenced in the United States for "worsening" relapsing remitting and secondary progressive MS. Dosage is limited by cumulative cardiotoxicity to a maximum of $120 \mathrm{mg} / \mathrm{m}^{2}$. We report experience from one centre on the use of this agent in patients with aggressive relapsing-remitting MS (RRMS).

Patients: 18 Patients have been treated $(11$ female), mean age 34.6 (range 20-47), mean disease duration 4.1 years. All patients had experienced two or more disabling relapses with incomplete recovery in the preceding year. Mean Expanded Disability Status Scale (EDSS) prior to treatment was 6.1. All patients underwent regular ECG and Echocardiography for left ventricular ejection fraction.

Results: There were no major adverse events, only one patient elected to discontinue treatment (at 18 months, disability stable). Mean duration of treatment was 17 months. In the 2 years prior to treatment the cohort had 60 steroid treated relapses (ARR 1.66), on treatment there were six relapses (ARR 0.35). At most recent follow up, or withdrawal of treatment, EDSS was stable (+/20.5 points) in 11 and improved (=1 point) in seven (mean EDSS 5.0).

Conclusions: Although we would acknowledge that the data we report is uncontrolled; in patients with very active RRMS mitoxantrone seems to be highly effective in suppressing relapse activity. The drug is well tolerated over periods of up to 24 months. Further studies examining the use of Mitoxantrone as an 'induction' agent in patients with aggressive disease, prior to the use of standard disease modifying drugs, should be considered.

\section{INTRATHECAL IMMUNE RESPONSE AGAINST AXONAL CYTOSKELETAL COMPONENTS IN PATIENTS WITH MULTIPLE SCLEROSIS: A PROGNOSTIC STUDY}

Seidi OA, Semera YK, Gregson NA, Sharief MK GKT School of Medicine, London, UK

Background: We have recently reported a significant correlation between clinical disability and intrathecal release of antibodies against axonal cytoskeleton in patients with primary and secondaryprogressive MS

Objectives: To determine the prognostic value of intrathecal antibodies to axonal proteins for future development of disability in MS patients.

Methods: In a prospective study, we measured intrathecal levels of antibodies against actin, tubulin and neurofilament light-subunit from 51 patients with relapsing-remitting, secondary, or primary progressive MS. Patients were clinically monitored for 2 years after the lumbar puncture (LP). We included 52 patients with other neurological disorders and eight healthy subjects, as controls. We also measured antibodies against myelin basic protein (MBP) as a control antigen.

Results: Intrathecal levels of antibodies against all axonal proteins, but not anti-MBP, correlated with the EDSS score at the time of LP. Moreover, MS patients with high intrathecal levels of antibodies against axonal proteins accumulated more neurological disability at the end of the two years period compared to patients with low levels. In contrast, antibodies against MBP did not correlate with EDSS score at the end of the study.

Conclusion: These findings suggest that the determination of intrathecal antibodies against axonal proteins may be clinically useful in MS patients. 


\section{OLIGODENDROCYTES PROMOTE THE SURVIVAL OF AXONS IN VITRO}

Wilkins A, Chandran S, Layfield R, Compston DAS Cambridge Centre for Brain Repair, Cambridge, UK

Background: Late onset axonopathy, occurring in the absence of inflammation, is an increasingly recognised feature of multiple sclerosis. The pathophysiology of this process however remains unknown, but loss of trophic support provided by oligodendrocytes, which are depleted in chronically demyelinated plaques, is one potential cause. We have therefore investigated oligodendrocyte influences on neurons and specifically on axons.

Methods: Rat neurons were cultured in oligodendrocyte conditioned medium (OCM) or non-conditioned medium (NCM). At determined time points, the cultures were stained with antibodies against axon specific proteins and axon length measured. Cell signaling analyses were carried out by western blotting.

Results: Neurons conditioned in OCM showed approximately $50 \%$ increase in axon length per neuron compared to those cultured in NCM. Furthermore inhibition of MAPkinase/Erk intracellular signalling pathway, which was activated by OCM, reduced this effect significantly.

Discussion: We have shown that soluble factors released by oligodendrocytes increase the survival of axons in culture, which may have important implications in determining the causes of axonopathy in multiple sclerosis. The identification of neurotrophic factors produced by oligodendrocytes may also lead to therapeutic strategies to prevent irreversible axon loss in late stages of the disease.

\section{A 5 YEAR LONGITUDINAL MRI STUDY OF VENTRICULAR VOLUME IN PRIMARY PROGRESSIVE MULTIPLE SCLEROSIS}

Ingle GT, Stevenson VL, Leary SM, Miller DH, Thompson AJ Institute of Neurology, London, UK

Background: Progressive atrophy resulting from axonal loss is one of the proposed mechanisms underlying disability in Primary progressive multiple sclerosis (PPMS). In this lengthy study of PPMS we measure ventricular changes over 5 years.

Methods: 41 PPMS patients representing a wide range of ages and disease duration attended for MRI and clinical examination at baseline, 1, 2 and 5 years. 2D T1 weighted images were used to measure ventricular volume at each time point using a semi-automated iterative morphological technique.

Results: Over 5 years there was a significant increase in ventricular volume $(p<0.0001)$ and the mean rate of change, by linear regression, was $1.14 \mathrm{ml}$ per year. Despite considerable variation in rate between patients (from 0.6 to $6.6 \mathrm{ml}$ per year), change over time appeared relatively constant for a given patient. There was no correlation between rate of ventricular enlargement and change in physical disability but an association was found with deteriorating cognitive function $(r=0.34 p=0.034)$

Discussion: In conclusion, ventricular enlargement is a sensitive measure of change in PPMS and demonstrates clinical validity with respect to cognitive function. It may also identify PPMS patients with early high ventricular enlargement rates who would benefit from therapeutic intervention.

\section{TEN YEAR SURVIVAL DATA FROM THE SCOTTISH MND REGISTER}

Forbes RB, Colville S, Parratt J, Chancellor A, Davenport R, Swingler RJ The Scottish MND Register, Ninewells Hospital, Dundee, UK

Aim: to describe survival from MND using a 10 year populationbased disease register.

Methods: survival analysis of Scottish adults with MND diagnosed between 1989 and 1998, using time-dependent cox regression.

Results: 1226 cases were registered, $54 \%$ were male and the mean age at onset was 64.7 years. 1054 cases $(86 \%)$ had been assessed by a neurologist. The first 614 cases were classified using the revised WFN criteria, and the El Escorial criteria have been used subsequently. The overall mean and median survival from symptom onset was 33.5 (SD 22.9) and 24.7 (IQR 15.6-40.8) months respectively. There was no influence upon survival of sex or family history. Shorter survival was associated with bulbar symptoms at onset $(\mathrm{HR}=1.25(95 \% \mathrm{Cl} 1.08-1.44))$, older age at onset $(\mathrm{HR}=1.02(95 \% \mathrm{Cl}$ $1.01-1.03)$ ) or presence of both upper and lower motor neuron signs at registration $(H R=1.22(95 \% \mathrm{Cl} 1.02-1.47))$. Improved survival was significantly associated with longer duration from symptom onset to diagnosis $(\mathrm{HR}=0.38(95 \% \mathrm{Cl} 0.33-0.42))$, assessment by a neurologist $(\mathrm{HR}=0.58(95 \% \mathrm{Cl} \quad 0.42-0.79))$ and treatment with Riluzole $(\mathrm{HR}=0.24(95 \% \mathrm{Cl} 0.14-0.40))$. Even allowing for different diagnostic criteria, there was a decline in median survival of 3 months between the first (1989-93) and second (1994-98) half of the study period.

Conclusions: survival from MND is explained by commonly identified clinical variables. There is a strong association between assessment by a neurologist and increased survival. The unanticipated decline in survival identified in the second half of the cohort remains unexplained.

\section{NEUROLOGICAL AND COGNITIVE DYSFUNCTION IN "NEVER-ENCEPHALOPATHIC" PATIENTS AWAITING LIVER TRANSPLANTATION}

Lewis MB, Stroud A, Davies M, Howdle PD St James's University Hospital, Leeds, UK

Introduction: Patients with liver failure who are not clinically encephalopathic can show evidence of neuropsychological impairment. Inadequate treatment of previous hepatic encephalopathy is often blamed. Repeated episodes of hepatic encephalopathy (HE) are thought to underlie the acquired hepatocerebral degeneration syndrome $(\mathrm{AHCD})$

Method: Patients listed for liver transplantation with no history of hepatic encephalopathy were assessed using a battery of neuropsychological tests and a structured neurological examination. Twenty five healthy volunteers were used as controls.

Results: Twenty patients were included. There were no significant differences between baseline characteristics of patients and controls. Highly significant global impairment of cognitive function was detected, with memory and visuospatial problems being prominent. No abnormalities were detected with the commonly used trail making test. Twelve patients had an abnormal neurological examination displaying many of the physical signs associated with $A H C D$.

Discussion: Cognitive and neurological dysfunction occurs in patients with end stage liver failure in the absence of previous HE. The impairment that occurs is severe, and affects many aspects of cognitive function. This has implications for the preoperative counselling of these patients and for obtaining informed consent. Current methods of monitoring cognitive impairment clinically, such as trailmaking tests, may be inadequate.

\section{ARE VERTEBROBASILAR TERRITORY TIAS AND MINOR STROKES LOWER RISK THAN CAROTID TERRITORY EVENTS?}

Flossmann E, Rothwell PM Stroke Prevention Research Unit, Radcliffe Infirmary, Oxford, UK

Vertebrobasilar (VB) TIAs and minor strokes are perceived to have a better prognosis than carotid events, and are sometimes managed less aggressively. However, this notion stems mainly from a few small studies in the 1960s and 70s, which did not report overall rates of vascular death, and it has never been systematically studied.

We reviewed all published studies of prognosis following a TIA or minor stroke using MEDLINE and EMBASE, and by hand searching reference lists and relevant journals. 820 abstracts were reviewed, and 303 papers were read in detail. 43 studies reported outcomes by territory of presenting event. Odds of recurrent events were calculated within studies and combined by fixed effects meta-analysis.

Patients presenting with $V B$ events had a lower risk of recurrent stroke than those with carotid events $(\mathrm{OR}=0.7,95 \% \mathrm{Cl} 0.6-0.8$, $\mathrm{p}<0.00001,33$ studies), but a higher risk of recurrent TIA (OR=1.9, $95 \% \mathrm{Cl} 1.5-2.4, \mathrm{p}<0.00001,12$ studies), and the same risk of death (mainly vascular) (OR=1.0,95\% Cl 0.8-1.2, $\mathrm{p}=0.95,17$ studies). Patients presenting with events in both territories had a higher risk of stroke than those with carotid events alone $(O R=2.0,95 \% \mathrm{Cl} 1.3-3.1$, $p=0.02$, six studies) or $V B$ events alone (OR=2.9, $95 \% \mathrm{Cl} 1.8-4.8$, $\mathrm{p}=0.0008$, six studies). There was no significant heterogeneity between studies for any of these results, and no differences between prospective and retrospective studies or studies with different diagnostic criteria.

Compared with patients presenting with carotid TIA or minor stroke, patients with VB events have a slightly lower risk of recurrent stroke, but the same risk of death and approximately twice the risk of TIA. There is no evidence to justify less aggressive preventive treatment. 


\section{HAEMODYNAMIC DETERMINANTS OF CAROTID PLAQUE INSTABILITY}

Lovett JK, Rothwell PM Stroke Prevention Research Unit, Radcliffe Infirmary, Oxford, UK

Plaque rupture is the main cause of acute coronary events and an important cause of ischaemic stroke. Haemodynamic forces, such as shear stress and pulse pressure (PP), are important in early plaque formation, but little is known about their effects on mature plaque. To determine whether haemodynamic forces affect plaque stability we studied the relationships between plaque rupture and the various components of blood pressure (BP - PP, DBP, SBP, MAP), and between the direction of blood flow (and hence the site of maximum shear stress) and the site of plaque rupture.

We studied angiograms of 3007 recently symptomatic carotid stenoses from the European Carotid Surgery Trial. We have shown previously that assessment of angiographic ulceration is reproducible and predicts stroke. The presence and position (relative to the point of maximum stenosis) of any ulceration was assessed, and correlated with the components of BP after correction for stenosis, age, sex, diabetes and smoking.

More ulceration occurred in the proximal ( $v$ distal) part of the plaque, where shear stress is highest $(\mathrm{OR}=7.2,95 \% \mathrm{Cl}=5.6-9.5$, $\mathrm{p}<0.001)$. PP was the strongest independent predictor of ulceration (OR per $\mathrm{SD}=1.20,95 \% \mathrm{Cl}=1.05-1.39, \mathrm{p}=0.01$ ). The relationship was weaker for SBP and MAP, and absent for DBP (OR per $S D=1.08$, $95 \% \mathrm{Cl}=0.94-1.25, \mathrm{p}=0.21$

Haemodynamic forces, such as shear stress and PP, do appear to be important in the rupture of mature carotid plaque. These results suggest that, in patients with large vessel disease, treatment of BP should take particular account of PP.

\section{IMAGING UNSTABLE CAROTID PLAQUES WITH ${ }^{18}$ FLURODEOXYGLUCOSE POSITRON EMISSION TOMOGRAPHY}

Warburton EA, Rudd JHF, Fryer TD, Jones HA, Gillard JH, Antoun NA, Kirkpatrick PJ Weissberg PL, Addenbrooke's Hospital, Cambridge, UK

Background: There is increasing evidence that rupture of carotid plaques causing transient ischaemic attacks (TIAs) and is the consequence of plaque formation. Current imaging techniques provide mainly structural information about the degree of carotid stenosis. The glucose analogue ${ }^{18} \mathrm{~F}$-flurodeoxyglucose $\left({ }^{18} \mathrm{FDG}\right)$ is taken up by activated inflammatory cells and can be used to image such activity by positron emission tomography (PET). This study used this form of imaging to identify inflammation in patients with symptomatic carotid stenotic plaques

Patients, methods, and results: Eight patients with carotid territory TIAs awaiting endarterectomy were imaged using ${ }^{18} \mathrm{FDG}$-PET. Images were coregistered to computed tomography (CT). Six of the eight patients had contralateral asymptomatic lesions. Symptomatic carotid plaques were clearly visible in ${ }^{18} \mathrm{FDG}$-PET images acquired three hours post ${ }^{18} \mathrm{FDG}$ injection. The net ${ }^{18} \mathrm{FDG}$ accumulation rate (plaque/integral plasma) in symptomatic lesions was on average $1.66310^{25} \pm 20.35310^{25}\left(95 \% \mathrm{Cl} 0.77-2.55310^{25}\right)$ higher than in contralateral asymptomatic lesions $(p=0.005)$. There was no measurable ${ }^{18} \mathrm{FDG}$ uptake into normal carotid artery (artery/plasma ration $1.0 \pm 20.1$ ). Autoradiography of excised plaques confirmed accumulation of ${ }^{3} \mathrm{H}$-deoxyglucose in macrophage-rich areas of the plaque.

Conclusions: This study demonstrates that ${ }^{18} \mathrm{FDG}$ is taken up into carotid plaques and that symptomatic unstable plaques accumulate significantly more ${ }^{18} \mathrm{FDG}$ than asymptomatic lesions. This may herald a new approach to carotid imaging that reflects that cellular pathology of atheroma rather than its anatomical consequences.

\section{INHIBITION OF TRIGEMINOVASCULAR NOCICEPTIVE TRANSMISSION BY A, RECEPTOR AGONISTS IN THE CAT}

Goadsby PJ, Hoskin KL, Storer RJ, Edvinsson L, Connor HE Institute of Neurology, London, UK

Introduction: Interest in the fundamental mechanisms underlying headache, particularly the pathophysiology of migraine and cluster headache, has led to the study of the physiology and pharmacology of the trigeminovascular system. The triptans, 5-HTIB/1D agonists, were substantial advances for many patients.
They have both vasoconstrictor and neuronal mechanisms of action. The literature suggests that adenosine receptor agonists may have anti-nociceptive effects without any vasoconstrictor action. We sought to explore any potential role in trigeminovascular nociceptive transmission.

Methods: Cats were anaesthetised (a-chloralose $60 \mathrm{mg} / \mathrm{kg}$, intraperitoneally and, in addition, halothane for all surgical procedures). The superior sagittal sinus (SSS) was isolated from underlying cortex and stimulated electrically after the animals had been paralysed with gallamine $(6 \mathrm{mg} / \mathrm{kg}$, intravenously). Units linked to stimulation were recorded with a tungsten-in-glass microelectrode placed in the trigeminocervical complex. Signals from the neurons were amplified, filtered and passed to a microcomputer for analysis. Blood was sampled from the external jugular vein to determine levels of calcitonin gene-related peptide (CGRP) release before and after drug administration.

Results: Units responded to SSS with a typical latency of 8-10 ms. Intravenous administration of the highly selective adenosine $A_{1}$ receptor agonist GR79236 (3, 10, 30 and $100 \mathrm{mg} / \mathrm{kg})$ had a dose-dependent inhibitory effect on SSS evoked trigeminal neuronal activity. The maximal effect was seen at $100 \mathrm{mg} / \mathrm{kg}$ with an $80 \pm 6 \%$ reduction in probability of firing. The inhibitory effect could be substantially reversed by the selective $A_{1}$ receptor antagonist DPCPX $(300 \mathrm{mg} / \mathrm{kg}$ iv; $p<0.05)$. SSS stimulation increased cranial CGRP levels from $33 \pm 2 \mathrm{pmol} / \mathrm{l}$ to $64 \pm 3 \mathrm{pmol} / \mathrm{l}$, an effect blocked by pretreatment with $30 \mathrm{mg} / \mathrm{kg}$ iv GR79236 (44 $\pm 3 \mathrm{pmol} / \mathrm{l}, \mathrm{n}=6$; $p<0.01)$.

Conclusion: It can be concluded that in two models of trigeminovascular nociceptive activation after SSS stimulation the $A_{1}$ receptor agonist GR79236 showed potent inhibitory activity. These studies suggest the adenosine $A_{1}$ receptor as a novel, non-vasoconstrictor target for the development of acute antimigraine treatments.

\section{DROPPED HEAD SYNDROME: THE DIFFERENTIAL DIAGNOSIS}

Nicholas RS, Lecky BRF The Walton Centre for Neurology and Neurosurgery, Liverpool, UK

Dropped head syndrome (DHS) is a disorder presenting with focal failure of neck extension that is usually found in elderly patients. The aetiology is variable with both primary neuromuscular and extra-pyramidal causes.

We describe 12 patients that presented with DHS who were investigated over a period of 5 years. The age of onset was 64 years (range 52-78 years), eight were women and four men. The eventual diagnoses after full investigation included: myotonic dystrophy (one patient), polymyositis (three patients), myopathy with rimmed vacuoles (one patient), myasthenia gravis (two patients), paraneoplastic motor neuronopathy (one patient), motor neuron disease (two patients), multisystem atrophy (one patient) and probable progressive supra-nuclear palsy (one patient). Of the three patients with polymyositis, two had concurrent malignancies all were successfully treated with steroids. Both patients with myasthenia gravis responded to steroids.

In all of these cases of DHS a precise diagnosis was made. Even in the elderly this syndrome is worth investigating as many have treatable pathology.

\section{HEALTH, DISABILITY AND DEATH: A NEW SPECTRUM OF DISEASE FOR HOMOPLASMIC MUTATIONS OF} MITOCHONDRIAL DNA

McFarland R, Taylor RW, Chinnery PF, Lightowlers RN, Turnbull DM University of Newcastle upon Tyne, UK

One of the cardinal features ascribed to pathogenic mutations of mitochondrial DNA (mtDNA) is that of heteroplasmy, a situation where both wild type and mutant copies of mtDNA coexist within a cell or tissue. Notable exceptions include the mutations causing single sensory organ impairment such as Leber's hereditary optic neuropathy (LHON) and aminoglycoside induced deafness (AID). In both of these conditions, homoplasmic mutations (where all copies of the mtDNA are mutant) cause disease. To date, there have been no reports of homoplasmic mutations resulting in lethal multisystem disorders.

We describe three different pathogenic homoplasmic mutations in families from North East England. The dichotomous phenotype associated with both LHON and AID is starkly contrasted against these family histories of healthy individuals, neonatal and early childhood 
deaths and progressive neurodisability. All of these mutations occur in mitochondrial tRNA genes, fulfil other accepted criteria for pathogenicity and are supported by striking biochemical, histological and steady state tRNA data.

At present these homoplasmic mutations represent a small but significant proportion of patients and their contribution to mitochondrial disease should not be overlooked. Indeed, they raise questions regarding the true incidence of mtDNA disease in the population. Furthermore, explaining the mechanisms behind the extreme phenotype variability will enhance our understanding of the whole field of mitochondrial genetics.

\section{EARLY ONSET FAMILIAL ALZHEIMER'S DISEASE: MUTATION FREQUENCY IN 31 FAMILIES}

Janssen JC, Beck JA, Campbell TA, Dickinson A, Fox NC, Harvey RJ, Houlden H, Rossor MN, Collinge J Institute of Neurology, London, UK

Background: Three causative genes have been identified for autosomal dominant Alzheimer's disease (AD): the amyloid precursor protein (APP), presenilin 1 (PSEN1) and presenilin 2 (PSEN2) genes. In addition a risk gene for $A D$ has been identified: the apolipoprotein E gene.

Methods: A mutational analysis of these genes was performed in 31 probands with probable or definite AD from UK families with an age at onset $<61$ years.

Results: The median age at onset was 45 years (range: 33-60 years). Twenty three probands had family histories consistent with autosomal dominant inheritance. In 17 probands 16 PSEN 1 mutations were identified, including a novel three base pair deletion mutation which effectively deletes codon 167 (2.167) and seven novel missense point mutations (Y154C, I229F, F237L, L253V, C263F, R377M and G378V). In three probands mutations in APP were identified, including a novel mutation at codon 715 (V715A). Mutations in PSEN 1 and APP were present in $78 \%$ of autosomal dominant $A D$ and $25 \%$ of patients who did not fulfil criteria for autosomal dominant inheritance. Eleven patients did not have a pathogenic mutation in APP, PSEN1, or PSEN2, these probands were older than those in whom mutations were demonstrated $(54.0 \vee 44.0$ years: $p=0.001)$ and had an APOE e4 allele frequency of 0.72 .

Conclusions: Mutations in APP and PSENI account for the majority of early onset autosomal dominant $A D$. The patients without a causative mutation were significantly older and had a higher APOE e4 allele frequency than the general white population suggesting familial clustering due to the presence of this risk gene.

\section{THE EFFECT OF EPILEPSY ON THE BRAIN: FINDINGS OF A LONGITUDINAL COMMUNITY BASED QUANTITATIVE MRI STUDY}

Liu RSN, Lemieux L, Bell GS, Sisodiya SM, Bartlett PA, Sander JWAS, Shorvon SD, Duncan JS Institute of Neurology, London

Background: The question of whether epilepsy causes cerebral damage remains unanswered. We report the first community-based quantitative MRI study investigating the longitudinal effect of seizures.

Methods: Serial MRI brain scans were performed on 190 epilepsy patients, recruited prospectively from a local population of 207553. Patients with chronic active epilepsy (122), new onset seizures (68), and age matched controls (90) were scanned 3.5 years apart. Manual, semiautomated, and automatic regional brain volume measurements were performed on coregistered image pairs. The investigator was blinded to subject status and the chronological order of each scan pair. Longitudinal volume changes were studied in three age epochs $(<35,35-54,>54$ years)

Results: Controls aged 35-54 and over showed brain atrophy, primarily due to white matter loss. Increased rates of hippocampal atrophy were seen over 54 years. Six of 68 newly diagnosed and 24 of 122 chronic epilepsy patients demonstrated significant volume changes. Within group analyses revealed significant hippocampal, cerebellar, white matter and total brain volume losses in both patient cohorts. Seizure frequency, number of AEDs and epilepsy syndrome were not significantly correlated with brain atrophy.

Discussion: Aging exerts differential effects on regional brain volumes. Nine per cent of newly diagnosed patients and $20 \%$ of patients with chronic epilepsy had significant volume loss; however seizures do not inevitably lead to cerebral damage.

\section{THE WREXHAM AREA EPILEPSY PROJECT}

Leach JP, Bartolo R, Nicolson A, Smith DF Wrexham Maelor Hospital and The Walton Centre for Neurology, UK

An increasing proportion of patients with epilepsy are diagnosed by neurologists. However, there is a large reservoir of patients with this label who have never seen a specialist. Our aim was to describe a sample of such patients living in the catchment of a District General Hospital.

All adults with epilepsy, on treatment, not attending an epilepsy clinic, identified from the records of 34 practices in the Wrexham area, were invited to attend for review of their condition

The first 200 patients are reported. The misdiagnosis rates for those diagnosed by non-specialists and neurologists were $21.3 \%$ and $6 \%$ respectively. The remission rates in patients with partial and idiopathic generalised epilepsies were 55 and $50 \%$ respectively, the latter being poorer than expected. The proportion of patient's offered, and accepting, change of treatment were 63 and $42 \%$ respectively. Irrespective of seizure control many patients were seeking specific information.

Even in an area with an established epilepsy clinic there is considerable unmet need. More than $40 \%$ of patient's will accept specialist follow up and an additional $10-20 \%$ would benefit from nurse counselling. This cross sectional data should inform future service developments.

\section{CEREBRAL METABOLITE AND NEUROTRANSMITTER CONCENTRATIONS IN IDIOPATHIC GENERALISED EPILEPSY MEASURED WITH SHORT ECHO TIME PROTON MR SPECTROSCOPY}

Simister RJ, McLean MA, Barker GJ, Duncan JS Institute of Neurology, London, UK

Background: Glutamate is the most important excitatory transmitter and $\gamma$-amino butyric acid (GABA) the dominant inhibitory neurotransmitter in the neocortex. We have developed proton MR spectroscopy (MRS) methods for reliably measuring GABA and the glutamate plus glutamine signal complex (GLX) in vivo. We now report on measurement of these metabolites in patients with idiopathic generalised epilepsy (IGE)

Methods: 25 patients with a diagnosis of IGE and normal MRI imaging and 18 normal control subjects were studied. All scans were performed on a 1.5T GE scanner. One voxel was prescribed (approx $35 \mathrm{ml}$ ) in each frontal lobe for each subject. N-acetyl aspartate (NAA) and GLX were measured by performing short echo time PRESS localised MRS whilst GABA measurement was via our double quantum GABA filter.

Results: Comparison was made between IGE and control groups. The right $(R)$ and left $(L)$ voxels were considered separately. The main findings were low NAA (R: $p<0.01, L: p=0.08)$ and high GLX (R: $p=0.08, L: p<0.05)$ in the IGE group. No group differences were observed for GABA.

Discussion: This is the first MRS study of GLX and GABA concentrations in IGE. The major finding of increased GLX suggests that abnormality in glutamate regulation and leading to intracellular accumulation could be a factor in IGE. Low NAA may well reflect subtle levels of neuronal dysfunction, despite normal MRI.

\section{POSTERIOR MESIAL TEMPORAL LOBE EPILEPSY: A DISTINCT SYNDROME?}

Wieshmann UC, Denby CE, Eldridge PR, Chadwick DW, Roberts N University of Liverpool, UK

Background: We propose a posterior mesial temporal lobe epilepsy (TLE) syndrome that is indistinguishable clinically from anterior TLE, but less amenable to epilepsy surgery.

Methods: We compared the presurgical $\mathrm{Tl}$ weighted magnetic resonance images of a group of TLE patients who became seizure free after resection of anterior mesial temporal structures $(n=18)$ with a group of TLE patients who continued to have seizure after this operation $(n=13)$ using statistical parametric mapping.

Results: We found a reduction in grey matter density in the posterior part of the temporal lobe (the occipito temporal gyrus) in surgical failures. This abnormality was ipsilateral to the resection but beyond the resection margin.

Discussion: Posterior and anterior mesial TLE appear to have identical clinical, electrical and structural features including mesial temporal sclerosis and specific tests to identify patients with posterior mesial epilepsy may be necessary to avoid unsuccessful epilepsy surgery. 


\section{TRANSIENT MONOCULAR BLINDNESS DUE TO VASOSPASM: VIDEO RECONSTRUCTION OF THE RETINAL VASCULATURE DURING AN ATTACK}

Petzold A, Islam N, Plant GT Moorfields Eye Hospital; The National Hospital for Neurology and Neurosurgery; Institute of Neurology, London, UK

Background: Transient monocular blindness (TMB) may be due to vasospasm embolism or other factors affecting retinal perfusion. Retinal vasospasm responds to calcium channel blockers.

Methods: Case presentation

Results: A 25 year old male patient presented with a 3 day history with about 40 episodes of loss of vision in the left eye. Each episode started with a 30 seconds to 1 minute phase where the vision slowly dimmed away. After 1 to 5 minutes of complete blindness (with absence of the direct pupil light reflex) vision returned over about 30 seconds. Recovery was always complete. Fundus examination during an attack showed narrowing of the central retinal artery and branches, retinal pallor and very slow flow with "cattle trucking" in the retinal venules. Sequential photographs of the fundus were obtained immediately after the onset of such an attack. The photographs were used to construct an animation which demonstrates for the first time the dynamics of the retinal vasculature during $T M B$ due to vasospasm. The patient was treated with $80 \mathrm{mg} /$ day nifedipine and no further episodes occurred during the 6 months follow up.

Discussion: Retinal vasospasm should carefully be sought for during diagnostic investigation of patients with $T M B$, particular if the episodes occur frequently. If $\mathrm{TMB}$ continues to occur after antithrombotic treatment and there is no evidence of embolism a trial with Ca-channel blockers would be recommended before subjecting the patient to more invasive procedures.

\section{A SUCCESSFUL DOMICILLARY TREATMENT FOR PATIENTS WITH HOMONYMOUS HEMIANOPIA}

Pambakian ALM, Mannon SK, Hodgson TL, Kennard C Imperial College School of Medicine; Charing Cross Hospital, London, UK

Patients with homonymous hemianopia $(\mathrm{HH})$ are disabled because they cannot scan the visual world effectively. We have therefore devised a simple rehabilitation tool based on a visual search (VS) paradigm. We hypothesised that by training hemianopes to adopt more efficient eye movement strategies into their blind field, their searching will improve and their disability reduce. The equipment is uniquely portable, low cost and easy to deploy by therapists naïve to the technique of VS. Patients can therefore be treated at home, causing minimal disruption to their daily lives.

Twenty nine hemianopes (15 L, 14 R) were treated at home under supervision. They practised 6 VS paradigms in 20 daily sessions lasting 40 minutes over 1 month. We examined their response times (RT) before and after training to determine whether they develop compensatory eye movement strategies with improvements in their overall function, measured objectively using tasks representing activities of daily living (ADL), and subjectively using a visual disorder questionnaire.

Patients improved in VS by adopting one of three strategies: Most (22) showed shorter mean RT, (ANOVA $p<0.0001$ ). Three patients with high error rates (>20\%) showed longer RT which improved with training in a complex speed accuracy trade off, (ANOVA $p<0.0001$ ). Four showed no significant change in response time but had significant training effects with high level hemifield interactions.

These represent specific and robust training effects, with accompanying improvements in $\mathrm{ADL}$ performance and questionnaire scores, but no concomitant visual field enlargement.

\section{HIGH FREQUENCY SACCADIC OSCILLATIONS ARE GENERATED BY BRAIN STEM, NOT CEREBELLAR, NEURONS}

Leigh RJ, Ramat S, Zee DS, Optican LM Case Western Reserve University, Cleveland, Ohio; Johns Hopkins University, Baltimore; National Eye Institute, Bethseda, USA

Objective: To determine whether saccadic oscillations, such as opsoclonus, arise primarily from the brainstem or cerebellar circuits.

Methods: We measured visually guided saccades and saccadicvergence movements in a patient with a midline surgical lesion that included both fastigial nuclei, and in five age-matched control subjects, using the magnetic search coil technique. We tested a current model for saccade generation, which includes brainstem saccadic burst neurons, and feedback control via the fastigial nucleus.

Results: The patient's visually guided saccades showed bilateral, horizontal hypermetria, and prolonged deceleration phase, consistent with experimental bilateral inactivation of the fastigial nucleus. During combined saccadic-vergence movements, the patient and control subjects all showed small-amplitude, high-frequency $(20-35 \mathrm{~Hz})$, conjugate horizontal oscillations of the eyes during the vergence movement that followed the initial saccade. The model predicted that, since feedback control of saccade trajectory by the fastigial nucleus was impaired in the patient, the high-frequency conjugate oscillations were generated by excitatory and inhibitory brainstem burst neurons which are configured in positive feedback, with high gain for small movements.

Conclusions: These findings suggest that high-frequency saccadic oscillations, as well as pathological flutter and opsoclonus, are generated by local brainstem connections between burst neurons, and occur when omnipause neurons are inhibited.

\section{DETECTION OF ASYMPTOMATIC LACUNAR INFARCTS WITH DIFFUSION WEIGHTED MRI: A POTENTIAL SURROGATE MARKER OF DISEASE ACTIVITY IN ISCHAEMIC LEUKOARAIOSIS AND CADASIL}

O'Sullivan M, Rich PM, Barrick TR, Clark CA, Markus HS Division of Clinical Neuroscience, St. George's Hospital Medical School, London, UK

Background: Cerebral small vessel disease is an important cause of cognitive impairment. It is usually sporadic but also occurs in the autosomal dominant disorder CADASIL. Recurrent lacunar stroke contributes to disease burden but symptomatic events are relatively rare, making large numbers necessary for clinical trials. Surrogate markers may have a role in screening therapies for efficacy. Diffusion weighted imaging (DWI) is sensitive to acute ischaemia and differentiates acute from chronic lacunar infarcts. If asymptomatic lesions were more common than symptomatic lesions they would be a potentially useful surrogate marker, analogous to detection of enhancing lesions as a marker in MS

Methods: Fifty patients with sporadic small vessel disease and 19 with CADASIL underwent DWI. All had been asymptomatic for 3 months before imaging. DWI were screened by two raters; new lesions were confirmed as recent by a visible reduction of mean diffusivity on mean diffusivity maps.

Results: Recent ischaemic lesions were identified in four patients with sporadic small vessel disease $(8.0 \%)$ and two with CADASIL $(10.5 \%)$. Estimated lesion frequencies were one every 125-175 days in the sporadic group and one every 95-133 days in CADASIL.

Conclusions: DWI detects asymptomatic new lesions with sufficient frequency to suggest a role as a surrogate marker of disease activity in sporadic small vessel disease and CADASIL.

\section{DOES DIFFUSION WEIGHTED IMAGING ALTER MANAGEMENT IN PATIENTS WITH SUBACUTE MINOR STROKE AND TIA?}

Schulz U, Briley D, Meagher T, Molyneux A, Rothwell PM Stroke Prevention Research Unit, Radcliffe Infirmary, Oxford, UK

Diffusion weighted brain imaging (DWI) is used in acute stroke, but may also be useful in patients with minor stroke or TIA when seen several days or weeks after symptom onset. Although visualisation of an acute ischaemic lesion, and knowledge of the number of lesions, and their territory(s), could influence management, there are no published studies.

119 consecutive TIA clinic patients who were considered likely to have had a cerebral ischaemic event underwent T2-weighted and DWI MR scanning. Two independent observers (neurologist and neuroradiologist) first reviewed the T2 images and then both the T2 and DWI scans. We assessed the frequency and certainty with which acute lesions were noted, and how often DWI provided additional clinically useful information.

DWI showed a recent lesion in $37(60 \%)$ of 62 patients with minor stroke and $8(14 \%)$ of 57 patients with TIA. Among 44 patients with symptoms <2 weeks, DWI was positive in $18 / 27(67 \%)$ strokes and $3 / 17$ (18\%) TIAs. Among 75 patients presenting $\geqslant 2$ weeks, DWI was positive in $19 / 35(54 \%)$ strokes and 5/40 (13\%) TIAs. In $27(44 \%)$ patients with stroke and $8(14 \%)$ with TIA, DWI provided additional clinically useful information. Inter-observer agreement for the identification of acute lesions was higher on DWI than on T2 (kappa $=0.94 \vee 0.49, \mathrm{p}<0.01)$. 
DWI is often positive several weeks after a minor stroke or a TIA. Interobserver agreement for the identification of acute lesions is higher than on T2, and DWI frequently provides additional clinically useful information that changes management. This suggests that combined T2 and DWI should be the imaging protocol of choice for patients with subacute minor stroke or TIA.

\section{IMPAIRED SPATIAL MEMORY CONTRIBUTES TO UNILATERAL NEGLECT}

Husain M, Mannan S, Mort D, Hodgson T, Driver J, Kennard C Imperial College School of Medicine, Charing Cross Hospital, London, UK

Right hemisphere stroke patients with unilateral neglect ignore stimuli to the left in everyday life, as well as on bedside tests that require them to search for visual objects. Such left neglect may reflect a bias to direct attention rightwards. We hypothesised that neglect may also involve a failure to remember locations that have been searched before.

We monitored eye position during visual search in 24 right hemisphere stroke patients (eight with severe left neglect; eight with mild neglect; and eight without neglect), as well as 16 healthy control subjects. We probed spatial memory during search by asking subjects to press a response button whenever they found a new target. They were asked not to press if they refixated a target they had previously judged to be new.

Eleven neglect patients demonstrated significantly impaired spatial memory, recursively re-examining stimuli on the right and, critically, failing to remember previously searched locations. High resolution MRI demonstrated that impaired spatial memory was associated with damage to discrete locations in the inferior parietal and inferior frontal lobe.

The degree of neglect correlated significantly with the spatial memory impairment. Treatments that target the memory deficit may therefore offer opportunities for reducing the severity of neglect.

\section{PERIODIC PARALYSIS, MALIGNANT VENTRICULAR ARRHYTHMIA AND DYSMORPHISM (ANDERSEN'S SYNDROME): A SKELETAL MUSCLE POTASSIUM CHANNEL DISORDER}

Davies NP, Weber A, Mueller R, Hilton-Jones D, Chinnery PF, Hanna MG Institute of Neurology, London, UK

Background: Andersen's syndrome is a triad of periodic paralysis (hypo or hyper), malignant ventricular arrhythmias and dysmorphism. The syndrome is often overlooked as the dysmorphism is subtle and the ventricular arrhythmias may not become symptomatic until later in the disease course. Mutations in a voltage independent skeletal muscle potassium channel gene (KCNJ2) were recently shown to cause this disorder

Methods: We identified three families with clinical features consistent with Andersen's syndrome. In addition to detailed clinical examination each patient underwent neurophysiological and cardiological investigations. Molecular genetic analysis of each proband was performed by direct DNA sequencing of the entire coding region of KCNJ2. Control chromosomes (160) were screened using restriction digests.

Results: Each family harboured a new pathogenic point mutation within KCNJ2. In family A, an aspartic acid was substituted for a glycine at codon (G300D) in the carboxy terminal of the channel. Family $B$ and $C$ harboured mutations in the amino terminal (D78G, R67W).

Conclusion: Andersen's syndrome should be considered in any patient with hypo or hyperkalaemic periodic paralysis. The ventricular arrhythmias may post date the onset of the paralytic episodes by years but the ECG often shows a long QT interval early on in the disorder. We have identified 3 new disease-causing mutations in KCNJ2, demonstrating that DNA-based diagnosis is possible in this disorder. It is important to note that non-penetrance occurs in this disorder and there is a high de novo mutation rate.

\section{EVIDENCE FOR UNDERDIAGNOSIS OF MYASTHENIA GRAVIS IN THE ELDERLY}

Vincent A, Clover L, Buckley C, Grimley Evans J, Rothwell PM Institute of Molecular Medicine, John Radcliffe Hospital, Oxford; Radcliffe Infirmary, Oxford, UK

Background: Myasthenia gravis (MG) can be confirmed by a positive acetylcholine receptor (AChR) antibody test, but the diagnosis can be missed in older patients. We looked for evidence of underdiagnosis of MG in the UK.

Methods: We identified positive AChR antibody tests in the UK during 1997-99 from all UK centres registered with the European Quality Assurance Scheme (EQAS), and calculated the age and sex specific incidence. We tested sera from 1127 elderly individuals, from the Oxford region, aged $\geqslant 75$ years.

Results: From the UK data, we identified 3183 positive AChR antibody tests giving an incidence of 1.8 cases/100 000/year. In both sexes, age specific incidence rose sharply between the ages of 55 and 70 , plateaved at $\geqslant 70$ years, and then fell sharply above 80 years. In our elderly cohort, aged $\geqslant 75$ years, eight sera $(0.70 \%)$ were positive for AChR antibodies. Only one of these individuals had had a previous clinical diagnosis of $M G$.

Discussion: The plateau and then fall over 75 years in the age specific incidence, together with the high prevalence of positive AChR antibody in the $>75$ year olds, suggests that myasthenia gravis is indeed under-diagnosed in the elderly. The results highlight the need for a high index of suspicion for this treatable disease.

\section{CHARACTERISATION OF THE CELL DEATH PATHWAY IN A CELLULAR MODEL OF FAMILIAL MOTOR NEURON DISEASE}

Sathasivam S, Menzies FM, Cookson MR, Grierson AJ, Shaw PJ University of Sheffield, UK

Background: Motor neuron disease (MND) ia a rapidly progressive neurodegenerative disorder, primarily affecting the upper and lower motor neurons. Around 10\% of MND cases are familial, of which one fifth are associated with mutations in the gene encoding the free radical scavenging enzyme copper/zinc superoxide dismutase-1 (SOD1). There is mounting evidence that the final motor neuron cell death pathway in MND is a programmed mechanism of cell death resembling apoptosis. The aim of this study was to characterise the sequential activation of molecular components in the apoptotic cell death pathway in a cell culture model of motor neuron injury induced by the presence of mutant SODI under basal conditions and or oxidative stress.

Methods: Using a motor neuron cell line (NSC34) stably transfected with mutant or normal forms of human SOD1, or with a vector alone, we examined the cell death pathway under conditions of oxidative stress induced by serum withdrawal. Initially we have examined biochemical and morphological indices of apoptosis, including expression of phosphotidylserine at the cell surface, nuclear fragmentation, and activation of caspases, a group of protease enzymes which are molecular effectors of apoptosis.

Results: Under basal conditions, cells containing mutant G93A SOD 1, compared to cell transfected with normal SOD 1 or vector only, showed changes suggesting an increased propensity to apoptosis, including activation of caspase 9 ( $p<0.0001$ by two way ANOVA) and externalisation of phosphotidylserine. When subjected to oxidative stress, the presence of mutant SODI resulted in a greater than $70 \%$ fall in cell viability compared to cells transfected with vector only. In addition, there was significantly increased activation of caspase 3 in the presence of mutant SODI in cells induced to undergo oxidative stress compared to control cell groups $(p<0.0001$ by two way ANOVA).

Conclusion: The presence of mutant SODI causes increased expression of molecules known to play a role in apoptotic cell death and an increased likelihood of neuronal cell death in the presence of oxidative stress.

\section{PROTEOMIC ANALYSIS OF PROTEIN ALTERATIONS IN A CELL CULTURE MODEL OF FAMILAL MOTOR NEURON DISEASE}

Shaw PJ, Allen S University of Sheffield, UK

Background: Mutations in the gene encoding Cu/Zn superoxide dismutase (SOD1) account for $20 \%$ of cases of familial motor neuron disease (MND). There is evidence that motor neuron $(M N)$ injury results from a toxic gain of function of mutant SOD 1, but the precise mechanisms of cell specific neurodegeneration have not yet been identified. Identification of $M N$ protein changes occuring in the presence of mutant SOD1 is likely to shed light on important molecular mechanisms underlying cellular injury.

Aims: The aims of this study were to investigate the effects of mutant SOD 1 on expression levels and post-translational modification of cellular proteins in a cell culture model of familial MND, using a proteomic approach. 
Methods: A robust cell culture model of familial MND has been developed using a motor neuron cell line (NSC34) stably transfected to express normal or mutant forms (G93A, G37R) of human SOD1. Subcellular fractionation allows proteins in different cell compartments to be analysed and we have focused intitally on the cytosolic fraction. Cytosolic proteins from this cell model were subjected to two dimensional gel electrophoresis which allows proteins to be separated first on the basis of electric charge and then by molecular weight. Cells expressing mutant forms of SODI were compared to control cells expressing normal SOD1 or cells transfected with vector only. Protein spots were visualised by silver staining and changes in protein expression analysed using Phoretix 2D software. Protein spots that increased or decreased in the presence of mutant SODI compared to control cells at a confidence level of $p<0.05$ (using two tailed Wilcoxon nonparametric paired $t$ test) were selected for identification. Protein spots of interest were excised and analysed by MALDI-TOF mass spectrometry. The resulting peptide mass profile allowed protein identification using an MS-fit database search.

Results: Spots representing 750 cytosolic proteins could be reliably identified from the NSC34 cells. In the presence of mutant SODl, significant changes in the expression of nine proteins were identified. Four of these proteins showed a greater than twofold increase in expression and five proteins were down regulated. MALDI-TOF analysis combined with protein data base searching has to date allowed the identification of five of these proteins. Confirmation of the changed expression was obtained by undertaking western blotting with antibodies specific to the candidate proteins on homogenates of the NSC34 cells. The identified proteins fall into four categories and are involved in pathways likely to be highly relevant to motor neurone degeneration. These include proteins involved in nitric oxide metabolism; nucleotide metabolism; protein degradation pathways and cytosolic redox regulation.

\section{DETECTION OF MUSK ANTIBODIES IN SERONEGATIVE MYASTHENIA GRAVIS USING HUMAN MUSK AS ANTIGEN}

McConville J, Hoch W, Beeson D, Newsom-Davis J, Vincent A Institute of Molecular Medicine, John Radcliffe Hospital, Oxford, UK

Background: Seronegative myasthenia gravis (SNMG), in which acetylcholine receptor antibodies cannot be detected, constitutes $20 \%$ of generalised and $50 \%$ of ocular myasthenia gravis. We have recently discovered that the muscle specific kinase, MuSK, is a target for autoantibodies in SNMG. Using rat MuSK extracellular domains in an ELISA, we detected MUSK antibodies in $70 \%$ of generalised SNMG (Hoch et al 2001). It was possible that the rat MUSK ELISA lacked sufficient sensitivity to detect all MuSK antibodies.

Methods: To obtain human MuSK protein for further studies, we cloned human MUSK and expressed the native protein in the human embryonic kidney (HEK293) cell line. We used the fluorescence activated cell sorter to detect antibody binding to the cells.

Results: All SNMG samples positive for antibodies to rat MuSK were also positive with human MuSK but $30 \%$ of generalised SNMG patients continued to be seronegative for both AChR and MuSK antibodies. We did not detect MuSK antibodies in pure ocular MG.

Discussion: Our results confirm the presence of MuSK antibodies in up to $70 \%$ of SNMG patients. The target of the antibodies in the remaining SNMG patients needs to be defined.

\section{RANDOMISED DOUBLE BLIND TRIAL OF METHOTREXATE AND STEROIDS COMPARED WITH AZATHIOPRINE AND STEROIDS IN THE TREATMENT OF IDIOPATHIC} INFLAMMATORY MYOPATHY

Miller JAL, Walsh Y, Saminaden S, Lecky BRF, Winer JB University Hospital Birmingham, UK

Background: The idiopathic inflammatory myopathies are a group of immune-mediated disorders characterised by muscle weakness and lymphocytic infiltrate in striated muscle. Corticosteroids form the mainstay of treatment with immunosuppressive treatment frequently used as an adjunct to reduce the long-term requirement for steroids. No randomised controlled trials have compared different immunosuppressive treatments. Rheumatological practice favours the use of methotrexate while azathioprine is often used by neurologists.

Methods: We have undertaken a randomised double blinded placebo controlled trial in 28 patients with either polymyositis or dermatomyositis. Subjects received prednisolone plus either azathioprine $2.5 \mathrm{mg} / \mathrm{kg}$ daily or methotrexate $15 \mathrm{mg}$ weekly. The primary end point of the study was the change in hand held myometry readings at the end of one year. Secondary endpoints were changes in timed walks, final steroid dose, and side effects of treatment.

Results: These are given in the table.

Discussion: Azathioprine and methotrexate had equivalent efficacy as adjunctive treatment but the better side-effect profile of methotrexate would suggest this to be the preferred agent in the treatment of idiopathic inflammatory myopathy.

\section{HOW MUCH DO NEURORADIOLOGISTS AGREE IN THEIR ASSESSMENT OF ANGIOGRAMS OF ARTERIOVENOUS MALFORMATIONS OF THE BRAIN? IMPLICATIONS FOR MANAGEMENT}

Al-Shahi R, Pal N, Lewis SC, Bhattacharya JJ, Sellar RJ, Warlow CP Department of Clinical Neurosciences, University of Edinburgh, Edinburgh, UK; Institute of Neurological Sciences, Southern General Hospital, Glasgow, UK

Background: The vascular anatomy (angioarchitecture) of brain arteriovenous malformations (AVMs) demonstrated on intra-arterial digital subtraction angiograms is thought to influence their prognosis, and is used by interventionists to decide whether and how to treat an AVM. But are neuroradiologists consistent in their description of angioarchitecture?

Methods: Five experienced neuroradiologists independently reviewed a selection of 40 anonymised angiograms from the time of first-ever AVM diagnosis, on two separate occasions. They assessed 16 separate features on the angiograms. We measured intra and interobserver agreement with the kappa statistic (k) for nominal data (eg, presence of aneurysms), weighted $k$ for ordinal and discrete interval data (eg number of feeding vessels), and Bland and Altman analysis for continuous data (eg, nidus size).

Results: Intraobserver agreement was generally moderate to substantial with $95 \%$ confidence intervals ranging from fair to almost perfect. However, for every characteristic, interobserver agreement was less than intraobserver agreement. Interobserver agreement was generally slight to moderate, with $95 \%$ confidence intervals ranging from less than chance to almost perfect.

Discussion: This study urges caution if relying on AVM angioarchitecture to make management decisions, and demonstrates the need for robust, generalisable definitions - with good observer agreement-for future efforts to understand the prognosis and best treatment of AVMs.

\section{MECHANISMS OF IDIOPATHIC INTRACRANIAL HYPERTENSION IN VASCULITIS PATIENTS WITHOUT CEREBRAL VENOUS SINUS THROMBOSIS}

Everitt A, Newry S, Graham E, Plant G National Hospital for Neurology and Neurosurgery; Medical Eye Unit, St Thomas' Hospital, London, UK

An association between systemic lupus erythematosus (SLE) and intracranial hypertension is well recognised but rare. Cerebral venous sinus thrombosis is identified as the underlying cause in some patients, whilst in the majority the pathogenesis is poorly understood.

We present six patients with SLE and one with Wegener's granulomatosis (WG) who presented with symptoms due to idiopathic intracranial hypertension $(\mathrm{IIH})$. Five had known SLE but, in two, the

Table Abstract 44

\begin{tabular}{lllll}
\hline & $\begin{array}{l}\text { Patients with }>30 \% \\
\text { improvement in myometry }\end{array}$ & $\begin{array}{l}\text { Average improvement } \\
\text { in walk time }(\%)\end{array}$ & $\begin{array}{l}\text { Average final steroid } \\
\text { dose }(\mathrm{mg})\end{array}$ & $\begin{array}{l}\text { Patients with grade } \\
3 \text { or } 4 \text { WHO toxicity }\end{array}$ \\
\hline Azathioprine $(n=12)$ & 5 & 16 & 11 & 4 \\
Methotrexate $(n=16)$ & 7 & 30 & 11 & 0 \\
\hline
\end{tabular}


development of $\mathrm{IH}$ led to the diagnosis of SLE and WG, respectively. Four patients were receiving combined oral steroids and immunosuppressants at presentation. Most subjects had evidence of high disease activity (systemic symptoms, high autoantibody levels and inflammatory markers. Three had recent weight gain and two had elevated anti-cardiolipin antibodies. One patient had diffusely abnormal cerebral white matter on MRI, but none had venous sinus thrombosis. CSF constituents were normal except in the WG patient who had a mild CSF lymphocytosis and elevated protein. One SLE patient subsequently developed cerebral lupus and died suddenly. Post mortem examination revealed multiple small cortical and brainstem infarcts, but no vasculitis or small vessel emboli were noted. A further SLE patient was found to have microemboli on renal biopsy.

It seems likely that IIH associated with vasculitis is multifactorial and may involve processes such as thrombotic obliteration of vascular beds supplying arachnoid villi, and immune complex deposition in the choroid plexus. Insights into the putative mechanisms of IIH from these seven patients will be discussed.

\section{A COMPARISON OF THE EFFECTS OF CAROTID ENDARTERECTOMY AND CAROTID ENDOVASULAR TREATMENT ON BLOOD PRESSURE IN THE ACUTE POSTOPERATIVE PERIOD}

McKevitt FM, Sivaguru A, Gaines PA, Cleveland TJ, Beard JD, Channer KS, Venables GS Royal Hallamshire Hospital; Northern General Hospital, Sheffield, UK

Background: Acute haemodynamic instability has been documented after both carotid endarterctomy and carotid angioplasty and stenting. This can result in prolonged hospital admission or neurological complication. This study compares the effects of both procedures on blood pressure in the first twenty-four hours following treatment.

Methods: Patients were randomised to carotid endarterectomy (49) or endovascular treatment (55). A baseline 24 hour ambulatory blood pressure recording was taken prior to carotid intervention and repeated during the firstn 24 hours postprocedure. The number of patients experiencing episodes of hypotension or hypertension was documented.

Results: Episodes of hypotension were seen frequently in both groups, $176 \%$ in the endovascular group and $70 \%$ in the surgical group, $p=0.50$ ). The number of patients in each group who experienced a hypertensive episode was also similar, $115 \%$ in the endovascular group $v 12 \%$ in the surgical group, $p=0.65$ ).

Discussion: Blood pressure disturbances are common following both endovascular and surgical treatment of carotid artery stenosis however neither intervention offers a haemodynamic advantage in the first 24 hours postprocedure.

\section{ACUTE ONSET NEW VARIANT CREUTZFELD-JAKOB DISEASE IN A COMPULSIVE JELLY EATER}

Das R, Molloy J, Nisbet A Hurstwood Park Neurological Centre, Haywards Heath, UK

A case of variant CJD (tonsillar biopsy confirmed) is presented, exhibiting some interesting features which may shed new light on this enigmatic disease.

Clinical presentation: Our patient presented to her GP with depression. She returned 3 weeks later with sudden onset numbness and heaviness over the left side of her face. She was noted to have a microvesicular eruption on the upper lip bilaterally. Viral antibody titres were negative. Since presentation she has swiftly deteriorated physically and cognitively. Dietary history revealed a habit of excessive consumption of jelly.

Investigations: Serial MRI scans show evolution of high T2 signal in the pulvinar and medial thalamus bilaterally, plus high signal in the medial geniculate bodies and superior colliculi.

Discussion: The case raises three interesting points for discussion: 1) could the rapid evolution of sensory symptoms with accompanying vesicular eruption, with negative conventional serology, represent a seroconversion equivalent in variant CJD, related to prion protein load? 2) the sequential MRI changes have not been seen before in this distribution, and may represent a new neuroradiological phenotype in this disease. 3) the potential for gelatin to carry the infective particles may warrant closer attention-it remains a potential route of human exposure to BSE.

\section{IDENTIFICATION OF NEW PRION DISEASE SUSCEPTIBILITY LOCI}

Mead S, Mahal S, Beck J, Farall M, Fisher EMC, Collinge J Institute of Neurology, London, UK

A common human prion protein gene (PRNP) polymorphism (encoding either methionine or valine at codon 129) is a strong susceptibility factor for sporadic and acquired prion disease. We have analysed the PRNP locus for tightly linked susceptibility factors. 56 polymorphic sites have been identified within $25 \mathrm{~kb}$ of the PRNP open reading frame including sites within the PRNP promoter and PRNP 39 untranslated region. These have been characterised in $61 \mathrm{CEPH}$ families, demonstrating extensive linkage disequilibrium around PRNP and the existence of eleven major European PRNP haplotypes. Haplotype frequencies estimated in healthy UK controls were very similar to those deduced in the CEPH families. A common haplotype was overrepresented in patients with sporadic CJD. Using a log linear modelling approach to simultaneously model Hardy-Weinberg and linkage disequilibria, a significant independent association was found between a polymorphism upstream of PRNP exon 1 and sporadic CJD $(p=0.005)$, in addition to the strong susceptibility conferred by codon $129\left(p=2 \times 10^{-8}\right)$. Although our sample size was necessarily small, no association was found between these polymorphisms and VCJD or iatrogenic CJD, in keeping with their having distinct disease mechanisms. In addition, there was no evidence of a PRNP founder effect in the first reported geographical cluster of VCJD. We hope to use genetic susceptibility loci like PRNP and the recently identified vCJD resistance factor HLA-DQ7 to characterise the molecular pathways involved in prion disease and stratify the evolving epidemic of variant CJD.

\section{THE BRITISH AND DANISH FORMS OF FAMILIAL BRI DEMENTIA}

Plant GT, Braendgaard H, Revesz T, Vidal R, Ghiso J, Frangione B National Hospital for Neurology and Neurosurgery, London, UK and New York University Medical Centre, New York, USA

Two enigmatic dominantly inherited familial conditions have been shown to be due to different mutations in the same gene. One is the disorder described by Worster-Drought in Britain in the 1930s which he called familial pre-senile dementia with spastic paralysis. The second originates from Jutland and was described in 1981 by Stromgren as heredopathia ophthalmo-oto-encephalica. In the British family a point mutation in the BRI gene results in an amyloid precursor protein (ABriPP 277) which is 11 amino acids longer than the wild type BRI protein (BRI 266). In the Danish family a 10 decamer nucleotide duplication gives rise to ADanPP 277. In each disorder a 34 amino acid amyloidogenic peptide is formed (ABri and ADan); the N-terminal 22 amino acid sequences are identical whilst the C-terminal 12 amino acids are completely different. Any differences between the two disorders should be explained by different properties of ABri and ADan. The major differences are seen below the age of 40 when Danish cases have already developed cataract and deafness, features not seen in the British families. From age 40 dementia, ataxia and spasticity are seen in both. Pathologically both conditions show amyloid angiopathy, cerebellar and hippocampal plaque formation and neurofibrillary tangles. We now refer to these disorders as the British and Danish forms of familial bri dementia.

\section{NON-CONVULSIVE STATUS EPILEPTICUS: A PROFILE OF PATIENTS DIAGNOSED WITHIN A TERTIARY REFERRAL CENTRE}

Haffey S, McKernan A, Pang KA Royal Victoria Hospital, Belfast, Northern Ireland, UK

Fifty episodes of NCSE were diagnosed in 45 patients over a 3 year period. We reviewed each case for a history of GTCS prior to confirmation of NCSE, a previous diagnosis of epilepsy, the underlying cause and co-morbidity along with conscious level and radiological findings.

Of the 45 initial presentations, NCSE was suspected by the referring clinicians in 36 cases. 25 patients had a previous diagnosis of epilepsy, but less than half the patients were recorded as having had a preceding GTCS.

Eleven patients had a history of learning disabilities and out of the 25 patients with previously diagnosed epilepsy, nine were thought to be in NCSE at the time of an acute systemic infection. 
Although one third of the patients in our group were semi or unconscious, $15 \%$ were alert and able to cooperate with the EEG.

We concluded that NCSE can occur in patients with no known history of epilepsy and should be considered as a differential diagnosis in prolonged postictal status.

Clinicians should maintain a high index of suspicion even in patients who appear to be alert and cooperative.

\section{TILT TABLE TESTING REFERRALS FROM AN EPILEPSY CLINIC}

Razvi SSM, Pascual J, Smith PEM University Hospital of Wales, Cardiff, Wales, UK

Head-up tilt table testing (TTT) is commonly used to investigate undiagnosed blackouts. Over 36 months, 132 patients referred from the epilepsy clinic underwent TTT. The main indication was recurrent undiagnosed blackouts likely to be vasovagal syncope. We did not refer clinically definite vasovagal syncope. TTT involved passive upright tilt at 60 degrees for 45 minutes. Complete data were available on 128 patients $(51$ male) aged $14-80$ (mean 37.1 ) years. Three diagnostic groups were identified before knowledge of the TTT results.

Probable vasovagal syncope $(n=75)$. TTT was positive in $49(65 \%)$, but subsequent diagnostic changes were rare, even with a negative test $(n=4(5 \%)$, 1 with epilepsy, 3 with other disorders).

Possible vasovagal syncope $(n=34)$. TTT was positive in $16(47 \%)$ and the diagnosis subsequently changed in 12 (35\%) (6 epilepsy, 6 other disorders).

Epilepsy with possible vasovagal syncope $(n=19)$. TTT was positive in $6(32 \%)$ and prompted diagnostic change in $11(58 \%)$ (epilepsy excluded in two, syncope unlikely in 9).

Twenty seven of 45 patients $(60 \%)$ previously treated with antiepileptic drugs were considered not to have had epilepsy.

We conclude that TTT is useful in investigating undiagnosed blackouts, particularly for possible rather than probable vasovagal syncope.

\section{DELAYS IN THE DIAGNOSIS OF PSYCHOGENIC NON-EPILEPTIC SEIZURES}

Reuber M, Fernandez G, Baver J, Helmstaedter C, Elger CE University of Bonn, Germany

Purpose: The prevalence of psychogenic non-epileptic seizures (PNES) in the community has been estimated as $4 \%$ that of epilepsy. PNES mainly affect young adults. Whilst overall outcome in terms of independent living is poor, several studies have shown that it is better if the diagnosis is made early. Our study describes the time to diagnosis in 313 consecutive patients and analyses factors contributing to delay.

Methods: All patients in whom a diagnosis of PNES was established at the Department of Epileptology, Bonn, Germany, between 1991 and 2001 were considered for inclusion $(n=337)$. Time of manifestation, clinical details and results of investigations were retrieved from the records. Patients were excluded if the manifestation was not recorded $(n=24)$. Mean delays were compared using $t$ tests for independent samples. The relationship between continuous variables and diagnostic delay was examined using a Pearson correlation.

Results: PNES were diagnosed after a mean of 7.2 years (SD 9.3 years). Younger age, interictal epileptiform potentials anticonvulsant treatment were associated with longer delays. Other patient factors did not explain diagnostic delay.

Conclusion: Mean diagnostic delay of PNES was over 7 years. Patient factors did not explain the great variability of delay suggesting that physician factors played an important role.

\section{QUANTITATIVE T2 MAPPING DEMONSTRATES ABNORMALITIES IN LOCALISATION-RELATED EPILEPSY}

Rugg-Gunn FJ, Symms MR, Boulby PA, Barker GJ, Duncan JS Institute of Neurology, London, UK

Background: Quantitative T2 mapping has previously been used in the evaluation of the hippocampus in patients with temporal lobe epilepsy, and has been shown to confer additional sensitivity over qualitative assessment. The aim of our study was to test the hypothesis that
T2 mapping of the whole brain would identify regions of abnormality in patients with localisation related epilepsy.

Methods: We acquired whole brain dual echo fast FLAIR MR images from 30 healthy subjects, nine patients with acquired lesions, 14 patients with malformations of cortical development (MCD), and 30 patients with refractory partial seizures and normal conventional MRI. The T2 maps of each patient were compared on a voxel by voxel basis to the control group using SPM99.

Results: Significant increases in T2 signal were identified in all patients with acquired lesions, and in 12 patients with MCD. Abnormalities were also found in 12 of the $30 \mathrm{MRI}$ negative patients, 10 of which concurred with electroclinical data.

Discussion: T2 mapping was sensitive in identifying regions of acquired injury or MCD, in some cases detecting abnormalities in regions previously thought to be normal. The T2 abnormalities in the MRI negative patients could be caused by either aetiological factors, such as occult MCD or as a result of chronic seizures, for example, atrophy and gliosis. These results show that T2 mapping, analysed on a voxel by voxel basis, is a promising imaging method for the evaluation of patients with partial seizures and normal conventional MRI.

\section{A NOVEL LOCUS ON CHROMOSOME 2P 14-21 IN A BRITISH FAMILY WITH GENERALISED EPILEPSY AND FEBRILE SEIZURES}

Siddiqui A, Johnson M, Dixon PH, Davis M, Koepp M, Shorvon SD, Sander JWA, Gardiner RM, Duncan JS, Wood NW Institute of Neurology, London; Royal Free and University College Medical School, London, UK

A large British family was identified with 12 affected individuals that manifested a variety of childhood onset epilepsies. The phenotype in this family included absences, myoclonus, frontal lobe epilepsy and generalised epilepsy, with or without preceding febrile seizures. Many of the individuals have features consistent with idiopathic generalised epilepsy. Linkage analysis has been performed and excluded the known loci for Generalised Epilepsy and Febrile Seizures plus (GEFS plus) ie, GEFS 1 and 2 (sodium channel genes SCNIB and SCN1A on chromosome 19 and 2 respectively) and GEFS 3 (GABA(A) receptor gamma 2-subunit gene on chromosome 5). The other subunits for the $G A B A(A)$ and $G A B A(B)$ receptors were also excluded. A genome wide search was then performed. Linkage was found to marker D2S337, with a maximum lod score of 3.05 at $u=0$. The candidate interval was flanked by markers D2S391 and D2S296, defining a region of $23 \mathrm{cM}$. We have therefore identified a new locus for GEFS plus on chromosome 2 at $2 p 14-21$. This indicates further heterogeniety for the GEFS plus phenotype. Potential candidate genes in this region are $\mathrm{KCNK} 12$, a potassium channel gene and SLC1A4, a solute carrier involved in chloride transport.

\section{PRESENCE OF ANTI-GLUR3 ANTIBODIES IN RASMUSSEN'S ENCEPHALITIS}

Watson R, Lang B, Beeson D, Bermudez I Weatherall Institute of Molecular Medicine, John Radcliffe Hospital, Oxford, UK

Background: Rasmussen's encephalitis (RE), which normally presents in children, is a rare progressive neurological disorder in which an autoimmune aetiology has been implicated. It is characterised by focal motor seizures evolving after a variable period of time to produce a focal neurological deficit, namely a hemiparesis with additional sensory deficits and cognitive deterioration. Antibodies against the AMPA-sensitive glutamate receptor 3 (GluR3) have been detected in some RE patients. Here we investigate the prevalence of these antibodies in sera from 20 RE patients.

Methods: We have cloned a full length GluR3 from human cDNA library, subcloned into the mammalian expression vector pcDNA3.1 Hygro and expressed in HEK 293 cells through transient transfection. Anti-GluR3 antibodies were screened using western blotting of the HEK-GluR3 cell lysate and ELISA using GluR3 peptides. GluR3 mRNA injected into $X$. Oocytes was used to investigate the electrophysiological functionality of these antibodies.

Results: Out of 20 patients screened, one tested positive for antiGluR3 antibodies using western blotting. Two patients evoked unusual currents in patch-clamping experiments that are not seen in other RE patients or controls.

Discussion: The data suggests that in a few patients with RE, autoantibodies may have a pathogenic role in the disease. However an anti-GluR3 antibody mediated aetiology for RE should not be implicated until there has been a definitive screen for these antibodies. 


\section{THE MOLECULAR BASIS FOR ABERRANT GENE REGULATION IN FRIEDREICH'S ATAXIA}

Everett CM, Saveliev A, Webster Z, Wood NW, Festenstein R Hammersmith Hospital; Institute of Neurology, National Hospital for Neurology and Neurosurgery, London, UK

Homozygous expansion of a GAA trinucleotide repeat within the first intron of the frataxin gene causes Friedreich's ataxia. The basis for frataxin gene repression in vivo is unclear. GAA repeat expansions may repress gene expression through an effect on DNA chromatin packaging.

Genes placed close to tightly packaged repressive chromatin exhibit a characteristic variegated expression profile, where a proportion of cells that would normally express become silenced. A human CD2 1.3 (hCD2 1.3) reporter behaves in this manner in transgenic mice. If an expanded GAA repeat, linked to hCD2 1.3, acts to condense chromatin then expression of hCD2 from this transgene would variegate even when integrated on the chromosomal long arm. Here chromatin is usually relaxed and permissive for gene expression. Twelve hCD2 1.3(GAA) 200 transgenic mouse lines were generated. Expression of hCD2 on T lymphocytes was measured by flow cytometry. In lines where the transgene integrated on chromosomal long arms, expression variegated strongly. DNase I hypersensitivity analysis confirmed that in non expressing T lymphocytes, hCD2 1.3 regulatory sequences were converted to tightly condensed chromatin.

Therefore a novel mechanism of GAA repeat mediated gene silencing occuring at the chromatin level was identified. Understanding such molecular mechanisms may provide future therapeutic strategies.

\section{CLINICAL AND GENETIC CHARACTERISATION OF FAMILIES WITH TRIPLE A (ALLGROVE) SYNDROME}

Houlden H, Smith S, de Carvalho M, Mathias C, Blake J, Wood NW, Reilly $M$ Institute of Neurology, London, UK

Triple A (Allgrove) syndrome is characterised by achalasia, alacrima, adrenal abnormalities and a progressive neurological syndrome. Affected individuals clinically have between two and four of these relatively common problems; hence the diagnosis is often difficult in all but the classical presentation. The inheritance is autosomal recessive and most cases of triple A have no family history. The triple A gene was recently identified at this locus and mutations reported in families from North Africa and Europe. The majority of mutations were homozygous.

We have identified 20 families with the clinical features associated with the triple A syndrome. Sequencing of the triple A gene revealed five families had a total of nine compound heterozygous mutations and one family had two homozygous mutations; these changes were spread throughout the triple $A$ gene in exons $1,2,7,8,10,11,12$, 13,16 and the poly $A$ tract. Families were clinically characterised in detail. Those bearing mutations had the classical triple A syndrome of achalasia, alacrima, adrenal abnormalities and a progressive neurological syndrome. We identified a spectrum of associated neurological abnormalities in these cases, including pupil abnormalities, optic atrophy, autonomic neuropathy and distal motor neuropathy with severe bilateral ulnar nerve involvement. In these families we have made genotype-phenotype correlations.

\section{GIANT AXONAL NEUROPATHY IS CAUSED BY MUTATIONS IN THE GIGAXONIN GENE: CLINICAL, GENETIC, AND PATHOLOGICAL CHARACTERISATION OF THREE FAMILIES}

Houlden H, Roper H, Willis T, Schenone A, Di Battista C, Wood NW, Reilly M Institute of Neurology, London, UK

Giant axonal neuropathy (GAN) is a severe childhood onset autosomal recessive sensorimotor neuropathy affecting both the peripheral nerves and the central nervous system. Ultrastructurally, axons are distended by masses of tightly woven neurofilaments. Keratin intermediate filaments also seem to be altered as most patients present characteristic curly or kinky hair. Recently the Gigaxonin gene on chromosome 16q24 was identified as the cause of GAN.

We sequenced all 11 exons and flanking intronic sequences of the Gigaxonin gene in three families with childhood onset neuropathy, typical sural nerve biopsy appearance and tight curly hair. We identified novel mutations in the Gigaxonin gene in all three families. Two consanguineous Pakistani families from Birmingham had homozygous mutations. The first family with one affected individual had a non-sense mutation in exon 10. The second Pakistani family had two affected individuals with homozygous missense mutations in exon 1. The third family was from Italy and had compound heterozygous mutations in the Gigaxonin gene that both caused amino acid changes. All mutations were not present in controls. We are currently sequencing the Gigaxonin gene in other individuals and we are offering this on a research basis in potential cases.

\section{A PARADIGM FOR EXPLORING THE GENETIC CONTROL OF BRAIN DEVELOPMENT AND FUNCTION}

Mitchell TN, Free SL, Shorvon SD, Sisodiya SM Institute of Neurology, London, UK

Background: Identification of the genes involved in the control of human cerebral development is important in the investigation of neurodevelopmental disorders, such as epilepsy and learning disability.

We present an MRI paradigm to examine the effects of one such gene, PAX6. Absence of functional PAX6 protein causes lethal cerebral malformations. Murine models suggest heterozygous PAX6 loss of function mutations (haploinsufficiency) causes subtle malformations. The biological effect and severity of PAX6 mutations differ, with haploinsufficiency being most severe. Human PAX6 heterozygotes have aniridia, but the cerebral effects are yet undetermined. MRI can reveal subtle cerebral malformations, with quantitative analysis extracting further structural information not evident on visual inspection.

Methods: We performed qualitative and quantitative MRI analysis in 20 patients with PAX6 mutations (point mutation, C-terminal expansion, missense mutation) and 100 sex/age-matched controls.

Results: Nine of 13 with point mutations had an absent anterior commissure and intact corpus callosum (a novel malformation). The anterior commissure was present in all patients with other mutations in addition to the 100 controls. Twelve of 13 had hyposmia. Quantitative analyses of cerebral morphology and connectivity (callosal cross sectional area, white matter surface area) were also abnormal.

Discussion: Using MRI we demonstrated differential effects of different PAX6 mutations. Consequences of PAX6 haploinsufficiency may include disruption of cellular differentiation, neuronal migration and axon guidance, functions known from murine models to be influenced by PAX6. Our study shows PAX6 is a candidate gene for human cerebral, as well as ocular, maldevelopment and that MRI provides a method for examining neurodevelopmental regulators.

\section{BRAIN STEM DYSFUNCTION SPECIFIC TO MIGRAINE HEADACHE}

Bahra A, Matharu MS, Buchel C, Frackowiak RSJ, Goadsby PJ Institute of Neurology, London, UK

Introduction: Primary headache syndromes have been classically diagnosed and differentiated by a sound history. Positron emission tomography (PET) studies have shown a differential brain activation pattern in migraine and cluster headache, specifically, brainstem activation during migraine and hypothalamic activation during cluster headache. We report two single PET studies in patients with both migraine and cluster headache who experienced a migraine attack while in an active cluster period.

Methods: The patients were imaged with PET following nitroglycerine provocation. An $\mathrm{H}_{2}{ }^{15} \mathrm{O}$ bolus technique was used to examine changes in regional cerebral blood flow, as an index of synaptic activity. The data were analysed using Statistical Parametric Mapping '99.

Results: Both patients experienced a typical migraine headache fulfilling International Headache Society criteria despite being in an active cluster period. First, there was activation of structures generally reported in functional brain imaging studies of pain : the cingulate, prefrontal, posterior insular and cerebellar cortices, the thalamus and basal ganglia. Secondly, there was strong bilateral activation corresponding to the region of the large intracranial vessels, which resolved with treatment of the headache. Thirdly, there was lateralised activation of the rostral brainstem which persisted following treatment with sumatriptan, and headache resolution. There was no activation of the hypothalamus.

Conclusion: These data demonstrate attack phenomenology predicting the appropriate brain activation pattern and the fundamentally neurovascular nature of migraine headache. 


\section{A GENOME-WIDE SCAN OF 42 CANADIAN FAMILIES SUFFERING MIGRAINE WITH AURA}

Cader MZ, Noble-Topham SE, Mandalfino P, Wingerchuk D, Brown JD, Rice GPA, Weber JL, Ebers GC Radcliffe Infirmary, Oxford, UK

Background: Migraine is a common neurological condition affecting upto $18 \%$ of the population. Classic migraine is associated with an aura (MA) and epidemiological studies suggest a strong genetic component. Linkage analysis can be useful in identifying susceptibility loci in complex traits and we have performed a genome wide scan to identify any such loci in 42 Canadian families

Methods: Forty two families with MA were interviewed using a validated migraine questionnaire. Headache type was classified using the International Headache Society (IHS) criteria. A genome wide scan was performed using 366 autosomal microsatellite markers and the Marshfield Genotyping Service. Fastlink 5.01 was used for 2 point linkage analysis.

Results: We did not find any regions of significant linkage in the total population. Two loci on chromosomes 1 and 11 were suggestive of linkage and had LOD scores greater than 2.0. However some individual families gave scores above 2 .

Discussion: Linkage has recently been reported on 55 Finnish families to chromosome 4 with a 2 point Lod score of 3.75. In our study there was no evidence of linkage in this region. This supports MA being a heterogeneous disorder. Further analyses with a larger number of families may help elucidate the susceptibility loci.

\section{PROLACTINOMA AND DOPAMINE-AGONIST INDUCED HEADACHE}

Levy M, Matharu MS, Goadsby P Institute of Neurology, London, UK

Introduction: Headache is a common problem in patients with pituitary tumours. Small pituitary lesions can cause debilitating headache, and suggest that the size of the pituitary tumour may not be the only causal factor in pituitary-related headache. Dopamine has been proposed to be an important neurotransmitter in primary headaches, and report two cases of primary headache triggered by dopamine agonists. We describe the clinical course of two patients with pituitary tumours experiencing distinct headaches that were exacerbated by dopamine agonists.

Case 1: A 36 year old woman, with no headache history, presented in 1987 with secondary amenorrhoea, galactorrhoea and headache. Serum prolactin was $700(27-525) \mathrm{mU} / \mathrm{I}$ and MRI confirmed a microprolactinoma. The headache was described as a constant left-sided sharp 'knife-like' pain with during exacerbations. An exacerbation would typically last between 10 seconds and 4 minutes and would occur up to 20 times a day. Precipitants for exacerbations included bending, walking and moving about. In between these exacerbations, she had a continuous dull left sided pain. During an exacerbation, she experienced ipsilateral cranial autonomic symptoms of lacrimation, nasal stuffiness, and ptosis. The clinical diagnosis of short lasting unilateral headache attacks with conjunctival injection and tearing (SUNCT) was made. The headaches completely settled after pituitary surgery and returned when the tumour recurred. Treatment with cabergoline and quinagolide on separate occasions triggered prolonged and very severe episodes of pain.

Case 2: A 40 year old woman presented in 1997 with right sided throbbing headaches. She had experienced headaches during her menarche and has a sister with migraine. She had not experienced headache for over 20 years. The presenting headache was associated with nausea, vomiting, photophobia, and phonophobia. Movement would exacerbate the headache. A typical attack would last 3-4 days, and occurred every 2 weeks. During an attack, she experienced ipsilateral conjunctival injection and lacrimation. Concurrently, she developed secondary amenorrhoea; serum prolactin was $2300 \mathrm{mU} / \mathrm{I}$ amd MRI showed a microprolactinoma. On treatment with bromocriptine she developed daily headache that persisted after cessation of treatment. Eventually she responded to treatment with indomethacin. The second case has a clinical diagnosis of hemicrania continua.

Conclusion: In each case, the administration of dopamine agonists led to an exacerbation in symptoms. This suggests that the dopamine-prolactin axis may be important in understanding the pathophysiology of primary headache syndromes.

\section{GREY MATTER NEURONAL LOSS IN MULTIPLE SCLEROSIS}

Cifelli A, Wylezinska M, Esiri M, Palace J, Matthews P The Radcliffe Infirmary, Oxford; FMRIB Centre, Oxford, UK

Multiple sclerosis (MS) is viewed as a prototype of white matter disease. Axonal loss has been increasingly recognised as an important contributor to disability. We have investigated the involvement of grey matter and neurons in MS.

Thalamic: (a) volume with magnetic resonance imaging (MRI), (b) neuronal integrity with proton magnetic resonance spectroscopy (MRS), (c) neuronal numbers with histopathology techniques were assessed. Fourteen secondary progressive patients and 14 age matched healthy controls were studied with 1H-MRS and MRI. Neuronal numbers in the thalamic mediodorsal (MD) nucleus were obtained in postmortem specimens from $10 \mathrm{MS}$ cases and 10 non-neurological controls using histopathological techniques.

Thalamic: (a) volumes, (b) NAA concentrations (a) neuronal marker, and (c) total neuronal numbers, were all significantly lower in MS patients compared with controls.

Such neuronal loss in the grey matter could play a major role in the permanent disability and cognitive dysfunction of MS which have so far been attributed to white matter damage.

\section{TRIPLE DOSE GADOLINIUM ENHANCED MRI OF THE BRAIN AND SPINAL CORD IN EARLY RELAPSING REMITTING MULTIPLE SCLEROSIS IS PREDICTIVE OF FUTURE RELAPSES}

Davies GR, Chard DT, Griffin CMB, Rashid W, Thompson A, Miller DH Institute of Neurology, London, UK

Background: Triple dose gadolinium (Gd) enhanced MRI is more sensitive at detecting active multiple sclerosis lesions than MRI with standard dose $(0.1 \mathrm{mmol} / \mathrm{kg})$ enhancement. This study has assessed the ability of triple dose gadolinium enhanced MRI to predict subsequent relapse rate in a cohort of early relapsing-remitting multiple sclerosis (MS) subjects.

Methods: Thirteen subjects with symptoms of MS for less than 3 years were imaged at months $0,1,2$, and 3 with triple dose Gd enhanced MRI of the brain and spinal cord. Lesions that were identified as newly enhancing after baseline were used to calculate the mean enhancing lesion number for the three month period. Relapse rate was assessed every 6 months for 2 years.

Results: Mean enhancing lesion number for the first 3 months (cord and brain combined) correlated significantly (Spearman's coefficient $r_{s}=0.77, p=0.002$ ) with yearly relapse rate for the whole 24 month period.

Discussion: Given the strong correlation seen between enhancing lesion number and relapse rate it is likely that triple dose Gd enhanced imaging of the brain and spinal cord provides a better assessment of inflammatory disease activity than single dose Gd enhanced brain imaging alone. The homogeneous nature of the cohort could also have improved the correlation.

\section{A GENOME-WIDE SCAN FOR REGIONS SHARED IDENTICAL BY DESCENT IN HUTTERITE MS FAMILIES}

Dyment DA, Datta A, Steckley JL, Willer CJ, Sadovnick AD, Risch NJ, Hader W, Ebers GC University of Oxford; University of Saskatoon; Stanford University, USA; University of British Columbia, Canada

Background: The Hutterites are a religious group living throughout mid-western Canada and the United States. They have practiced a degree of endogamy for the last 100 years and as a result an individual Hutterite is related to another as a first cousin once removed. They have been a useful study group for the study of infertility and asthma.

The complexity of MS genetics has made the search for novel genes using sib-pair allele sharing methods difficult. To minimise the genetic heterogeneity thought to be present in the MS population, we screened the Canadian MS population for people belonging to the Hutterite Brethren.

Methods: In a screen of 18000 Canadian MS patients the CCPGSMS identified seven individual Hutterites with clinically definite multiple sclerosis. These seven MS patients are all related to a common founder couple by eight generations. We genotyped these seven people and their 22 family members for 400 markers. 
Haplotypes were then created and visually inspected to see which intervals were shared among the MS patients.

Results: There was no interval shared among six MS patients (one patient of the seven refused to have familiy members contacted). However there were five regions $(6 p 21,6 q, 14 q, 15 p, 17 p, 18 q)$ that were shared among five of the six Hutterites MS patients. The HLA Class II region was one of these five shared regions. The haplotype shared did not bear the DRB 1 * 15 allele but the DRB 1 *04 allele.

Discussion: No single region was shared between the Hutterite patients. This could be due to the resolution of the markers used or heterogeneity in MS pathogenesis in this Founder population. This strategy does work as the MHC was identified in the screen. Interestingly, the MS associated MHC haplotype in this population was not the $\mathrm{DRB} 1{ }^{*} 15$ allele but $\mathrm{DRB} 1{ }^{*} 04$, allele suggesting a situation perhaps more akin to that seen in Sardinia.

\section{CHARACTERISATION OF THE ASTROCYTIC GLIAL SCAR IN MULTIPLE SCLEROSIS}

Gutowski NJ, Holley JE, Gveric D, Newcombe J Royal Devon and Exeter Hospital, Exeter; Institute of Neurology, London, UK

Introduction: In the chronic plaques of multiple sclerosis (MS), astrocytic scarring inhibits tissue repair. Animal work has shown that the antigenic phenotype of the most abundant cell type in the brain, the astrocyte, varies depending on astrocyte type and location. Characterisation of human astrocytes in MS tissue may identify markers of the scarring seen in chronic lesions.

Methods: Astrocytic phenotype was investigated in sub-ventricular white matter by immunocytochemistry and western blotting. Snap frozen tissue from normal controls $(n=4), M S$ normal appearing white matter $(n=5)$ and lesions [acute $(n=7)$, sub-acute $(n=7)$ and chronic $(n=13)]$ was studied.

Results: As expected, glial fibrillary acidic protein, vimentin and tenascin- $C$ and $-R$ expression was elevated in areas of scarring. There was also increased expression of nestin, embryonic neural cell adhesion molecule, receptor nerve growth factor, nerve growth factor, and its receptor p75.

Conclusion: Changes in expression of several proteins have been found to characterise the scar astrocyte phenotype in chronic MS lesions. The role of proteins associated with development, growth factors and growth factor receptors in glial scar formation and tissue repair is the subject of further investigation.

\section{EVALUATING THE USE OF B-INTERFERON IN THE UK: FROM WHOSE PERSPECTIVE?}

Hobart J, Riazi A, Lamping D, Fitzpatrick R, Thompson AJ Institute of Neurology, London; Derriford Hospital, Plymouth, Devon, UK

The Department of Health have announced they intend to fund a scheme making $\beta$-interferon available for people with MS in the UK. Longitudinal data will be collected to evaluate change over time. We examined how the choice of rating scale could influence the results of that scheme.

Three groups of people with MS were studied: consecutive admissions for inpatient rehabilitation $(n=64)$ and IV steroids for relapses $(n=77)$, a cohort with primary progressive MS $(n=104)$. Multiple rating scales were administered to all samples at two points in time. Scales were physician-based leg. Expanded Disability Status Scale-EDSS, MS Functional Composite-MSFC, Guys Neurological Disability Scale-GNDS) and patient-based (eg, Multiple Sclerosis Impact Scale-MSIS-29, Short Form 36-SF36)

We examined the reliability and validity of each scale, correlations between physician and patient-based scales purporting to measure similar aspects of health, the ability of competing scales to detect change, the interpretation of change scores.

All scales satisfied standard reliability and validity criteria. Correlations between physician and patient-based scales varied 10.20 to 0.75 ). The ability of competing scales to detect change varied (effect size 0.10 to 1.0). The "effectiveness" of rehabilitation and steroids, as judged by statistical significance of change scores, was scale dependent. Significant changes were demonstrated with some patient-based scales (eg. MSIS-29, SF-36) but not some physician-based scales (EDSS, MSFC).

The outcome of the scheme will almost certainly be influenced by the choice of rating scale and the perspective of the rater.

\section{SERUM AUTOANTIBODIES AGAINST INTRACELLULAR ANTIGENS IN MULTIPLE SCLEROSIS}

Lily O, Palace J, Vincent A Radcliffe Infirmary, Oxford, UK

Multiple sclerosis (MS) is thought to be an organ-specific autoimmune disease. The hallmark of these disorders is the production of autoantibodies. In many cases, autoantibodies are directed against intracellular antigens. A number of intracellular antigens have been proposed for MS, including the heat-shock protein, alpha-B crystallin.

We tried to demonstrate autoantibody binding to intracellular antigens using sera from MS patients. Immunohistochemistry with rat brain stem tissue sections failed to show significant antibody binding in any of 40 MS patients. Flow cytometry of permeabilised human oligodendrocyte, astrocyte and neuronal cell lines failed to show any significant difference in antibody binding between $58 \mathrm{MS}$ patients and 28 controls. When the oligodendrocyte cell line was pretreated with heat shock, there followed a marked increase in antibody binding from MS sera. However, this was also seen with control sera. Heat shock had no effect on antibody binding to the astrocyte cell line.

We conclude that there is no evidence for autoantibodies against intracellular antigens in MS patients. Autoimmunity toward heat shock proteins may be a feature of inflammation and may account for the relative vulnerability of oligodendrocytes to inflammatory damage. If a specific autoimmune process underlies MS, then the autoantigen is likely to be a cell surface protein.

\section{MULTIPLE SCLEROSIS IN SOUTHEAST IRELAND: UPDATING THE PREVALENCE}

McGuigan C, McCarthy A, Hawkins S, Hutchinson M St Vincents University Hospital, Dublin, Ireland

Objective: To determine the prevalence of, and range of disability due to, multiple sclerosis (MS) in County Wexford in the Republic of Ireland.

Background: The south east of Ireland has been considered to have a relatively low prevalence of MS. Previous prevalence data has been published on County Wexford; Brady et al in 1971: 54.5 per 100000 and Hutchinson in 1984: 48.4 per 100000

Methods: Sources of case ascertainment were: a postal survey of general practitioners, county physicians and consultant neurologists; hospital coding lists; the local MS society; respite care facilities and interferon prescription lists.

Results: One hundred and twenty six patients with clinically definite or probable MS (Poser criteria) were identified from 161 referrals, a prevalence rate of 120.7 per $100000(95 \%$ confidence intervals $99.6-141.8$ per 100,000 ). Approximately $70 \%$ of these patients were individually assessed. The age range of prevalent cases is 19 to 73 years, mean age 47.5 years, and mean age at onset 35.6 years. Female to male ratio 2.2:1. Mean Kurtzke expanded disability score 4.36 (0-9.5); Multiple sclerosis functional composite score 21.281 (26.5535-0.7739); and multiple sclerosis physical impact scale score $37.54(0-85)$

Conclusions: County Wexford has a higher prevalence of MS than previously reported. This is unlikely to represent a true increase in prevalence but better case ascertainment, earlier diagnosis and increased survival.

\section{NO EVIDENCE TO SUPPORT CTLA4 AS A MULTIPLE SCLEROSIS SUSCEPTIBILITY GENE}

Morrison K, Dyment DA, Willer CJ, Risch N, Sadovnick AD, Ebers GC University of Oxford, UK; University of Stanford, USA; University of British Columbia, Canada

Background: The cytotoxic T lymphocyte antigen 4 gene (CTLA- 4 ) is involved in the regulation of $T$ cell proliferation. During $T$ cell activation, CTLA-4 is expressed on the surface of the T cell. It then binds to its B7 ligand on the antigen presenting cell (APC) and signals an end to the T cell proliferation response. As such, CTLA-4 serves as a candidate susceptibility gene for autoimmune disorders. Polymorphisms at the CTLA-4 locus have been shown to be associated with autoimmune disease such as Hashimotos thyroiditis, diabetes, Graves disease, and multiple sclerosis.

Methods: Two polymorphisms of the CTLA-4 gene were genotyped in 232 sibling pairs affected with multiple sclerosis (MS) from 185 families. The CTLA-4 polymorphisms genotyped were an 39 untranslated (AT) $n$ microsatellite and an alanine/threonine RFLP of exon 1 . 
Results: There was no evidence for linkage by either identify by descent or identify by state methods. A transmission disequilibrium test (TDT) was performed and no preferential transmission of alleles was observed. Upon stratification of patients, there was observed no preferential transmission based upon gender, by presence or absence of HLA*DRB ${ }^{*} 15$ by ethnicity or by clinical course of the disease.

Discussion: CTLA-4 does not appear to be a major MS susceptibility locus in Canadian multiplex families.

\section{THE UK MS DATABASE}

Palace J, Hoddell B, Boggild M, Rogers S The Radcliffe Infirmary, Oxford; The Walton Centre, Liverpool, UK

The UK has a high incidence of multiple sclerosis (MS) with an estimated 85000 cases, however, the number of neurologists - and specifically neurologists with an interest in MS-remains low by comparison to North American and northern European figures. Though the few expert clinicians in this field poses obvious difficulties, services for patients with MS have expanded in recent years - hand in hand with developments in disease modifying therapies. MS services are provided in different settings, ranging from academic centres with multidisciplinary teams to single handed neurologists within district general hospitals. Though a number of centres have basic, locally developed database systems, no common system for data collection is available, nor have more than a handful of centres become involved with the EDMUS database system.

The UK MS database represents an attempt to develop a bespoke but flexible MS database system to meet the varied needs of clinicians working with MS patients in the UK. The system was developed following consultation with representatives from more than 20 centres across the UK, with input from professionals across a range of disciplines, involved in the care of patients with MS. As a result of this combination, the database has been designed as a free standing system for local use on laptop or network systems.

The key features of the system are as follows:

Microsoft Access based system;

Minimal core data set;

Additional data modules which can be configured locally;

Multidisciplinary input;

Graphical displays of individual patient histories;

Flexible data analysis package;

Potential for data pooling via SQL server system.

Database coordinators have been employed to support the package within centres across the UK and the system will be subject to regular updates through a central user group. The database has completed final pilot testing in a variety of settings across the UK and is now freely available. The database will be demonstrated with the poster.

\section{THE COMPARATIVE INVESTIGATION OF CEREBRAL PERFUSION IN MULTIPLE SCLEROSIS USING A NOVEL TECHNIQUE}

Rashid W, Parkes LM, Ingle GT, Chard DT, Tofts PS, Miller DH Institute of Neurology, London, UK

Background: Cerebral perfusion in multiple sclerosis (MS) has not been previously estimated using continuous arterial spin labelling (CASL), an MRI technique using endogenously labelled arterial water. This is potentially more accurate than other techniques as it allows quantitative perfusion estimates with less susceptibility to contrast loss in areas of blood brain barrier disruption. This study compares perfusion measures between normal controls and subtypes of MS using CASL.

Methods: Thirty two normal controls and 34 MS subjects $(11$ primary progressive, 10 relapsing remitting (RR), eight benign, five secondary progressive (SP)) were compared using CASL.

Results: General linear modelling with age as a covariate and sex as a fixed factor, showed a significant increase in white matter perfusion in the $\operatorname{RR}(p=0.008)$ and SP $(p=0.026)$ groups. No significant changes were seen in the grey matter.

Discussion: Due to the small cohort sizes interpretation should be cautious, however this is the first study to show increased perfusion in white matter in RR and SP MS subjects. This may reflect increased inflammatory activity in these subgroups. This study suggests CASL may be of use in monitoring inflammatory activity in MS.

\section{AUDIT OF B-INTERFERON TREATMENT: EXPERIENCE IN THE FIRST 100 PATIENTS WITHIN THE WALTON CENTRE MS SERVICE}

Rog DJ, Young CA, Boggild MD Walton Centre for Neurology and Neurosurgery, Liverpool, UK

Aim-To ascertain whether patient selection for $\beta$-interferon ( $\beta$-IFN) side-effect profile and relapse outcome in early clinical practice is in accord with data from published randomised clinical trials (RCTs) in relapsing remitting MS (RRMS).

Methods: We performed a retrospective case notes audit of the first 100 patients in the Liverpool and Chester MS clinics treated with $\beta$-IFN. Pretreatment demographic details, MS history and Expanded Disability Status Scale (EDSS) were collected. $\beta$-INF preparation first prescribed, number of relapses, EDSS, adverse events on treatment, reason for withdrawal of $\beta$-IFN and subsequent alternative treatments were determined.

Results: Eighty one patients were female. Pretreatment variables were: 35 years (range 18-58, SD 8.06), duration of MS 7.7 years $(0.7-29,6.39)$, relapses in 2 years prior to treatment $3.1(1-7,1.08)$ and EDSS $4.4(1.5-6.5,1.3)$. Fifty patients received Rebif, 32 Avonex, and 18 Betaferon. Fifty eight patients remain on treatment (mean 36 months), 53 are "responders" (relapse free or EDSS stable), for all patients annualised relapse rates on treatment were reduced by $33 \%$. For the group of patients on treatment for at least 6 months, ie excluding early treatment failures $(n=85)$ reduction in relapse rate was $49 \%$. In those patients on treatment for a minimum of 12 months (74), the reduction was $61 \%$. Thirty one patients withdrew from treatment (mean 15.5 months) due to disease progression or relapses. Eleven withdrew due to adverse events (mean 5.9 months). Twenty four of those withdrawn from $\beta$-INF are receiving alternative disease modifying treatments.

Conclusions: Patients being identified for $\beta$-INF treatment broadly match those recruited into RCTs. Adverse events also mirror those from trials with an $11 \%$ drop out rate. The reduction in annualised relapse rate with time on treatment reflects selection of $\beta$-IFN "responders" and underlines the importance of operating treatment withdrawal guidelines.

\section{OVEREXPRESSION OF CD5 ON B LYMPHOCYTES CORRELATES WITH THE DISEASE ACTIVITY IN PATIENTS WITH RELAPSING-REMITTING MULTIPLE SCLEROSIS}

Seidi OA, Semra YK, Sharief MK GKT School of Medicine; Guy's Hospital, London, UK

Multiple sclerosis (MS) is regarded as a T lymphocyte mediated disease. There is emerging evidence that B lymphocytes also play a role in disease pathogenesis. A subtype of B lymphocytes, the CD5 expressing $B$ cells, are often increased in several autoimmune disorders. Therefore, we sought to examine the role of these cells in MS. Using flow cytometry, we studied the expression of CD5 on peripheral B lymphocytes from 28 patients with relapsing remitting MS (RRMS), 13 of whom had active MS according to clinical and MRI criteria. We also included 42 patients with other inflammatory and noninflammatory neurological disorders as controls. T cell expression of CD4 and CD8 was studied for comparison. We detected significantly high expression of CD5 on B lymphocytes from patients with active RRMS, but in only three controls with untreated cerebral lupus. There was no difference in the expression of the pan-B cell antigen CD19, or $T$ cell antigens CD3, CD4 and CD8. Within the active RRMS group, there were no correlation between CD5 and CD19, CD3, CD4 or CD8 expression. Our results suggest a possible pathogenic role for CD5 in active RRMS. Further studies to explore mechanisms of trafficking of these cells into the central nervous system might open avenues for a more targeted therapy of MS.

\section{FORTY MULTIPLEX FAMILIES WITH MS: LINKAGE ANALYSIS OF CHROMOSOME 6 AND HLA-DRBI}

Willer CJ, Dyment DA, Armstrong H, Risch N, AD Sadovnick, Ebers GC Univeristy of Oxford, UK; University of British Columbia, Canada; University of Western Ontario, Canada; Stanford University, USA

Background: Multiple sclerosis is a neurological, autoimmune disease that affects tens of thousands of Canadians. Family studies of half-siblings, adoptees, and siblings have shown that susceptibility is clearly determined by both genes and environment. 
Methods: We ascertained 18000 Canadian patients with multiple sclerosis and selected the 40 families that were most informative for linkage analysis. Epidemiological and clinical data was available from 187 of 252 affected individuals. Microsatellites on chromosome 6 and the HLA-DRB 1 locus were genotyped and linkage analysis was performed.

Results: Two point parametric, multipoint parametric and NPL analyses were performed There was suggestive but not significant linkage to the HLA-DRB 1 locus. No linkage to microsatellites in the $6 \mathrm{p} 21$ region was found. The results suggest there may be populations of families in which the DRB1 has little or no influence on susceptibility and others where it is important.

Discussion: Multiplex families have the potential to be more powerful and require smaller genotyping effort. However, linkage analysis methods are susceptible to misspecification of the inheritance patterns. Heterogeneity is an important consideration in linkage studies of large families of MS and its elucidation may require both genetic and epidemiological subgrouping.

\section{RECESSIVE INHERITANCE AND VARIABLE PENTRANCE OF SLOW CHANNEL MYASTHENIC SYNDROMES}

Beeson D, Croxen R, Hatton C, Shelley C, Colquhoun D, Chauplannaz G, Oosterhuis H, Newsom-Davis J, Vincent A University of Oxford; University College, London, UK

Background: Reported cases of slow channel congenital myasthenic syndrome (SCCMS) have been dominantly inherited disorders due to missense mutations in the muscle acetylcholine receptor (AChR). Single channel recordings show that all cause the prolonged activations of the AChR ion channel that underlies the disease pathology.

Methods: Three SCCMS families were screened for AChR mutations using single channel conformation polymorphism analysis, DNA sequencing and restriction endonuclease digestions. Functional effects of the mutations were analysed using single-channel recordings of mutant AChR expressed in HEK293 cells.

Results: We identify two new mutations in the AChR $\epsilon$-subunit gene, $\epsilon \mathrm{L} 78 \mathrm{P}$ and $\epsilon \mathrm{L} 221 \mathrm{~F}$. The mutations prolong ion channel activations 3.7 and 2.4 fold respectively. $\epsilon \mathrm{L} 78 \mathrm{P}$ was identified in a family in which symptoms only presented in the index patient, born to consanguineous parents, who is homozygous for the mutation. $\epsilon \mathrm{L} 221 \mathrm{~F}$ shows limited penetrance in one of the two families with this mutation.

Discussion: Differential diagnosis between SCCMS and AChR deficiency syndrome, which shows recessive inheritance, is important since they require contrasting treatment strategies. Here, we provide the first defined examples of recessive inheritance and variable penetrance for SCCMS. A diagnosis of SCCMS should not be ruled out in suspected cases of CMS that show a recessive inheritance pattern.

\section{ANTIBODIES TO FETAL ANTIGENS IN MOTHERS OF BABIES WITH ARTHROGRYPOSIS MULTIPLEX CONGENITA (AMC) OR OTHER NEURODEVELOPMENTAL DISORDERS}

Dalton P, Clover L, Beeson D, Vincent A Weatherall Institute of Molecular Medicine, John Radcliffe Hospital, Oxford, UK

Background: Arthrogryposis multiplex congenita (AMC) is a non-progressive disorder in which babies are born or die in utero with multiple fixed joint contractures. It can be caused by any condition which leads to restriction of fetal movement. Occasionally women with myasthenia gravis (MG) have children with AMC due to high titres of antibodies against the fetal isoform of the acetylcholine receptor (AChR). However, the frequency of maternal antibody-mediated AMC is unknown.

Methods: We tested sera from more then 200 women who have had one or more babies with $A M C$ or who recurrent pregnancies affected by other developmental disorders. We used in vitro techniques (radioimmunoassay, flow cytometry, ELISA, immunohistochemistry, western blotting) on rhabdomyosarcoma and neuroblastoma cell lines and on human fetal and newborn mouse tissues to look for maternal antibodies.

Results: We found fetal AChR antibodies in $2 \%$, and antibodies to other muscle, neuronal or chondrocyte antigens in a further $12 \%$. The unknown antigen(s) involved are being defined by $\mathrm{N}$-terminal sequencing and screening of cDNA expression libraries.

Discussion: The results suggest that AMC and other developmental disorders can be associated with maternal antibodies against fetal antigens; further work is needed to define the targets and demonstrate antibody mediated pathogenicity.

\section{THE SODIUM CHANNEL SYNDROMES: EXPANDING THE PHENOTYPE ASSOCIATED WITH SCN4A MUTATIONS}

Davies NP, Sutton I, Winer JB, Moorcroft P, Pall HS, Cole T, Davies MB, Valente EM, Brancati F, Hammans SR, Hanna MG Institute of Neurology, London, UK

Background: Paramyotonia congenita, potassium aggravated myotonia and hyperkalaemic periodic paralysis (hyperPP) are allelic disorders caused by mutations in the skeletal muscle voltage gated sodium channel gene (SCN4A). Certain mutations within this gene are associated with one of the three disorders but it is now emerging that several mutations may cause an overlap syndrome leg, paramyotonia with hyperPP).

Methods: We identified 15 families with either paramyotonia congenita alone or paramyotonia congenita plus hyperPP Detailed clinical and neurophysiological examination (including cold immersion EMG) was performed in each case. Molecular genetic analysis of SCN4A was performed with a combination of restriction fragment length polymorphism analysis and direct DNA sequencing.

Results: Ten of the 15 probands harboured a mutation in SCN4A. We identified new phenotypes in association with three known mutations. Four families with paramyotonia/hyperPP overlap harboured the T704M (3) or M1592V(1) mutations previously associated with isolated hyperPP. In two families with paramyotonia/hyperPP we identified an $\mathrm{R} 1448 \mathrm{H}$ mutation formerly associated with isolated paramyotonia. We identified a new mutation in exon 24 in one family (L1435P) associated with isolated paramyotonia.

Conclusion: Overlap syndromes are common in the skeletal muscle sodium channel disorders. Exon 13 (T704M) and exon 24 (R1448H, M1592V) seem to be hotspots for mutations resulting in paramyotonia/hyperPP overlap. We have identified a new mutation in exon 24 confirming that this exon and particularly the region encoding the voltage sensor of domain IV is a hotspot for mutations causing paramyotonia congenita.

\section{USE OF PEPTIDE: HLA CLASS II COMPLEXES TO STUDY SPECIFIC T CELLS IN MYASTHENIA GRAVIS}

Kishore U, Zhang W, Corlett L, Kelleher A, Shiono H, Willcox N Weatherall Institute of Molecular Medicine, John Radcliffe Hospital, Oxford, UK

The pathogenic autoantibodies in MG depend on specific helper T cells. They recognise acetylcholine receptor (AchR) derived peptide epitopes when presented by HLA-class II molecules on antigen presenting cells. These $T$ cells appear much less heterogeneous than the antibody producing $B$ cells, and therefore more suitable for specific immuno targetting

We have recently identified two recurring AChR epitopes that are presented by HLA DR52a (DRB3*0101) to specific T cells cloned from several early onset MG patients (Hill et al, 1999). To assess their potential dominance and pathogenicity rapidly in a wider range of patients and controls, we are using soluble peptide linked DR52a ectodomains attached to an $\lg G 2 a$ Fc framework. When these lgG-like dimers are cross linked by fluorescent protein-A, the resulting multimers bind much more avidly to our cloned T-cells' receptors (which have low affinities for monomeric ligands). They give low backgrounds on control blood T cells; we are now staining blood and thymic cells from patients.

Soluble peptide:class II complexes are versatile reagents: monomers can also induce tolerance if cultured with T cells in the absence of antigen presenting cells, by engaging the T cells' receptors without costimulation. We are now comparing monomers, dimers, and multimers, aiming to generate reagents suitable. 


\section{NOVEL MUTATION IN THE MUSCLE ACETYLCHOLINE RECEPTOR A-SUBUNIT UNDERLIES A FAST CHANNEL CONGENITAL MYASTHENIC SYNDROME}

Webster R, Vincent A, Newsom-Davis J, Beeson D Weatherall Institute of Molecular Medicine, John Radcliffe Hospital, Oxford, UK

Background: Congenital myasthenic syndromes (CMS) are frequently caused by mutations in the muscle acetylcholine receptor (AChR). The mutations may result in reduced receptor number and/or kinetic abnormalities that alter AChR function.

Methods: AChR subunit genes were screened for mutations using single strand conformation polymorphism analysis, DNA sequencing and restriction endonuclease digestion. Effects of mutations on the function of AChR expressed in HEK293 cells were determined by cell attached patch-clamp single channel and burst analysis.

Results: Mutational analysis identified one missense mutation, aF256L, present in the index patient and his father. Analysis of mutant AChR activity showed very brief burst durations typical of a "fast channel". The longest population of bursts for the aF256L-AChR had a mean duration of $1.87 \pm 0.49$ ms compared with $3.62 \pm 0.61 \mathrm{~ms}$ in wild-typeAChR. In addition, the proportion of all bursts in the longest population was greatly reduced $(1.98 \pm 0.8 \%$ for aF $256 \mathrm{~L}$ vs. $28.2 \pm$ $3.9 \%$ for wild-type).

Discussion: Reported cases of the fast channel syndrome have shown recessive inheritance. Since we were unable to detect a second pathogenic mutation, and a biopsy from the patient's father showed evidence of dysfunction in neuromuscular transmission, this CMS phenotype may result from a "dominant negative" effect of the ?F256L mutation

\section{ATYPICAL MUSCLE BIOPSY FINDINGS IN A PATIENT WITH 4Q35 FACIOSCAPULOHUMERAL MUSCULAR DYSTROPHY}

Wood-Allum C, Brennan P, Hewitt M, Lowe J, Tyfield L, Wills A University Hospital Queen's Medical Centre; Nottingham City Hospital; University of Nottingham Medical School, Nottingham, UK; Southmead Hospital, Bristol, UK

Background: Facioscapulohumeral muscular dystrophy (FSHD) is a common, autosomal dominant myopathy. It is usually the result of deletions at $4 q 35$. Current diagnostic criteria for FSHD exclude patients with atypical muscle biopsy findings. The 64 year old man presented here had clinical features typical of FSHD and no family history. His muscle biopsy, however, had features specific to nemaline myopathy, a largely autosomal recessive disorder, whose clinical characteristics overlap with FSHD. When three of his children began to develop a similar syndrome the diagnosis was reviewed and genetic testing for FSHD was carried out. All four family members were found to carry a $4 \mathrm{q} 35$ deletion. Whilst clinically atypical $4 \mathrm{q} 35$ carriers have been reported, this family suggests that the histopathological spectrum of FSHD also may be broader than is currently acknowledged.

Methods: An EcoR1/Bln 1 double digest technique was used to test for FSHD. The VNTR probe $\mathrm{pH} 30$ was used on a Hindlll digest of genomic DNA to establish that all three children had inherited the same paternal chromosome 4 allele.

Results: Muscle biopsy of the index case featured eosinophilic inclusions in $\sim 10 \%$ of muscle fibres, identified by electron microscopy as nemaline rods and plentiful enough to strongly suggest a nemaline myopathy. All four affected family members, however, were subsequently shown to have a shortened EcoR l digestion fragment of $17 \mathrm{~kb}$, confirming the presence of a $4 q 35$ deletion and the diagnosis of FSHD.

Discussion: Patients with no family history or evidence of an autosomal dominant myopathy in whom an FSHD-like syndrome is suspected should be considered for FSHD gene testing even if their muscle biopsy suggests an alternative diagnosis. Clinicians should in particular consider testing for FSHD in juvenile and adult onset nemaline myopathies. As cases accumulate of $4 \mathrm{q} 35$ carrying exceptions to currently accepted diagnostic criteria for FSHD, these may require modification.

\section{SODIUM CHANNEL PLASTICITY IN PAINFUL DIABETIC NEUROPATHY}

Craner M, Black JA, Waxman SG VA Medical Centre, Yale University, USA

Objectives: Diabetes mellitus (DM) is now the leading cause of peripheral neuropathy worldwide of which $11-25 \%$ of patients report pain. Accumulating evidence indicates that dysregulated sodium channel gene transcription leads to hyperexcitability of injured dorsal root ganglion (DRG) neurons, which can contribute to neuropathic pain. We examined sodium channel mRNA and protein expression in DRG to gain insight into the pathophysiology of allodynia in the streptozotocin model of diabetic neuropathy.

Methods: DM was induced in Sprague-Dawley rats with streptozotocin $(60 \mathrm{mg} / \mathrm{kg}$, i.p.). Allodynia was defined as a withdrawal threshold of $<4 \mathrm{~g}$ by Von Frey hair assessment. In situ hybridisation and immunohistochemistry assessed levels of mRNA and protein respectively for sodium channels $\mathrm{rNa}_{\mathrm{v}} 1.3, \mathrm{rNa}_{\mathrm{v}} 1.6, \mathrm{rNa}_{\mathrm{v}} 1.8$ and $\mathrm{rNa}_{\mathrm{v}} 1.9$

Results: There was a significant up regulation of $\mathrm{rNa}_{v} 1.3, \mathrm{rNa}_{v} 1.6$ and $\mathrm{rNa}_{\mathrm{v}} 1.9$ and a down regulation of $\mathrm{rNav} 1.8$ mRNA. Immunohistochemistry demonstrated parallel changes at the protein level.

Conclusions: Sodium channel gene expression in DRG neurons is dysregulated in an animal model of DM, and this may lead to hyperexcitability and neuropathic pain. Through greater understanding of the pathophysiology of neuropathic pain we can hope to identify specific therapeutic targets.

\section{CHRONIC IDIOPATHIC AXONAL POLYNEUROPATHY AND GLUCOSE TOLERANCE}

Hughes RAC, Umapathi T, Carey L Guy's, King's and St Thomas' School of Medicine, London, UK

We report on 33 patients from an ongoing investigation of chronic idiopathic axonal polyneuropathy (CIAP). In the evaluation we placed emphasis on elucidating possible family history, neurotoxic exposure, occult alcoholism, undiagnosed malignancy and abnormal glucose handling.

The mean (SD) age of the patients was 66.4 (8.9) years. Three quarters were male. The most common clinical subtype $(45 \%)$ was painful, predominantly small fiber sensory neuropathy. The other subtypes were painless sensorimotor $(21 \%)$, painful sensorimotor neuropathy $(6 \%)$ and predominantly motor neuropathy $(18 \%)$. One patient, with a painless predominantly motor sensory neuropathy, has a similarly affected sibling

Twenty five per cent of all CIAP and $47 \%$ of those with painful sensory neuropathy had abnormal OGTT with normal glycosylated haemoglobin. Two patients, had glucose levels diagnostic of diabetes mellitus, four had impaired glucose tolerance and two impaired fasting glycaemia as defined by $\mathrm{WHO}$. All but one had painful sensory neuropathy.

In summary CIAP is heterogeneous but the proposed clinical subtypes delineated may define more homogeneous entities. In support of this hypothesis, about half of CIAP patients have a painful, predominantly small fibre sensory neuropathy in which impaired glucose tolerance may be an aetiological factor.

\section{THE RISK OF RELAPSE OF GUILLAIN-BARRÉ SYNDROME OR CHRONIC INFLAMMATORY DEMYELINATING POLYRADICULONEUROPATHY FOLLOWING IMMUNISATION}

Pritchard J, Mukherjee R, Hughes RAC Guy's, King's and St Thomas School of Medicine, London, UK

Background: The safety of immunisation after recovery from Guillain-Barré syndrome (GBS) or in the related condition chronic inflammatory demyelinating polyradiculoneuropathy (CIDP) is not known. Patients and physicians often avoid immunisation for fear of precipitating relapse.

Methods: We audited the occurrence of self reported relapse in a postal survey of 3000 members of the GBS Support Group. We relied upon patients' own reports of their underlying condition and relapses, confirming the latter by telephone discussion or by consultation with their neurologist.

Results: After immunisation, eleven out of 311 GBS patients and five out of 65 CIDP patients noted recurrent neurological symptoms. None of the GBS patients were hospitalised or treated. In two of the CIDP patients the deterioration was sufficient to cause a temporary increase in the modified Rankin scale score from 1 to 4

Discussion: The method of data collection may have overestimated the incidence of relapse rates after immunisation. Nevertheless we can conclude with 95\% confidence that in GBS the risk of relapse after immunisation requiring treatment or hospitalisation is less than $1.2 \%$. In CIDP the upper $95 \%$ confidence limit of the risk of recurrent symptoms following immunisation was $17 \%$, which may in part reflect the natural history of the disease. 
86 CABERGOLINE MONOTHERAPY IN CLINICAL PRACTICE: A TWO YEAR OBSERVATIONAL STUDY OF TOLERABILITY AND EFFICACY IN YOUNG, OLD AND VERY ELDERLY PATIENTS WITH PARKINSON'S DISEASE

Appiah-Kubi LS, Nisbet A, Burn DJ, Ray Chaudhuri K King's College Hospital; University Hospital, Lewisham; GKT School of Medicine; Royal Sussex County Hospital; Newcastle General Hospital, UK

Background: Cabergoline (CBG), the longest acting dopamine agonist, provides physiological sustained stimulation of dopamine receptors. We have reported efficacy of cabergoline in young and elderly Parkinson's disease (PD) patients, yet concerns over presumed side effects have limited the use of CBG as monotherapy., particularly in the elderly.

Methods: Patients on $C B G$ monotherapy $\left(\mathrm{CBG}_{\mathrm{m}}\right)$ were identified from a database of 299 PD patients, from three regional movement disorder centres. Records were assessed for demographic details and tolerability was defined as at least 6 months sustained CBG therapy.

Results: CBG monotherapy was identified in 53 patients, [mean age 62 (40-81); young ( $n=33)$ 55.03; elderly 73.5]. Mean CBG dose $(3.89 \mathrm{mg})$ and mean disease duration (3.96 years) was comparable in young and elderly. No dyskinesias were observed during a mean CBG treatment period of 26 months (range 2-54 months). $13.2 \%$ required additional non-levodopa PD therapy, while two patients, intolerant to other agonists were maintained on CBG $47.2 \%$ of patients reported subjective improvement in sleep disturbance. Side effects were uncommon.

Discussion: $C B G$ is well tolerated in a significant subset of young and elderly PD patients, with benefits including an exceptionally low rate of dyskinesia and improvement in sleep symptoms.

\section{AUTOSOMAL DOMINANT PARKINSONIAN SYNDROME CAUSED BY A MUTATION IN THE FERRITIN LIGHT POLYPEPTIDE GENE}

Hadjikoutis S, Walls TJ, Llewelyn JG Royal Gwent Hospital, Newport, Wales, UK

There is increasing evidence that iron-mediated abnormalities in the basal ganglia may play an important role in the pathogenesis of some parkinsonian syndromes, such as idiopathic Parkinson's disease, through free radical formation.

Ferritin is an important protein for intracellular storage and detoxification of iron. We present the case of a 70 year old woman with dominantly inherited parkinsonian syndrome and a mutation in the gene encoding ferritin light polypeptide. She presented at the age of 30 with progressive gait difficulty characterised by festination and recurrent falls. On examination her voice was hypophonic and speech slightly dysarthric. She had a fixed grimace/smile. There was marked nuchal rigidity and bilateral limb rigidity and bradykinesia with occasional upper limb rest tremor. Reflexes were symmetrically exaggerated but both plantars flexor. Muscle strength was normal and there was no wasting. She demonstrated normal limb praxis. Swallowing, eye movements and limb co-ordination were normal. She had quite marked postural instability. Her father, one paternal uncle, two paternal aunts, a paternal grandmother and her son had a similar parkinsonian syndrome indicating autosomal dominant inheritance. A trial of L-dopa up to $1000 \mathrm{mg}$ provided no benefit.

Linkage analysis confirmed a mutation in the gene encoding ferritin light polypeptide (chromosome 19q13.3) in all the affected members of the family. There was an adenine insertion at position 460-461 that is predicted to alter carboxy-terminal residues of the gene product. Full blood count, serum iron and ferritin and cearuloplasmin were all within normal range.

Mutations of proteins essential in iron metabolism should be considered in families with neurodegenerative disorders presenting with a progressive parkinsonian syndrome of unknown aetiology.

\section{LONG TERM SURVIVAL OF PORCINE EXPANDED NEURAL PRECURSORS IN THE RAT MODEL OF PARKINSON'S DISEASE}

Harrower T, Ratcliffe E, Richards A, Dunnett S, Barker R The Cambridge Centre for Brain Repair, UK

Background: Porcine embryonic tissue has been considered as an alternative source of cells for neural transplantation. However, such tissue is readily rejected as a result of the host immune system. Porcine expanded neural precursors (PENPs) or porcine stem cells may be resistant to immunological rejection and integrate into host tissue better than primary porcine fetal neural tissue.

Methods: Hemi-parkinsonian rats were transplanted with $1 \times 10^{\circ}$ cells prepared freshly from the cortex of E26 porcine embryos $(n=14$ and neurospheres containing $1 \times 10^{6}$ PENPs $(n=16)$ derived from the E26 cortex cultured for 21 days. Reduction in amphetamine-induced rotation was assessed as a measure of graft function. Graft survival and integration was assessed by histological analysis of the transplants.

Results: Grafts of PENPs survived better at each time point, with $100 \%$ of grafts surviving at 19 weeks compared to $40 \%$ in the primary tissue group. PENP grafts had significantly fewer lymphocyte and macrophage infiltrates. PENP grafts had significantly better fibre outgrowth and synapse formation. No significant reduction in rotation was detected

Conclusions: These results indicate that PENPs elicit less host immune response, are able to integrate significantly better into the host tissue and can survive for longer than 19 weeks.

\section{SUPPRESSION OF COMPLEX TREMOR IN MULTIPLE SCLEROSIS BY STIMULATION OF ZONA INCERTA: USE OF FIELD POTENTIALS}

Nandi D, Parkin SG, Gregory RP, Joint C, Stein JF, Aziz TZ Radcliffe Infirmary, Oxford, UK

Tremor is a common, disabling feature of multiple sclerosis that is usually refractory to medical therapy. Lesioning or chronic deep brain stimulation (DBS) of the lateral thalamic nuclei (VIM/VOP) can improve distal tremor in the contralateral limbs. These targets are less effective in controlling the complex limb tremor often seen in MS, which has proximal components and elements of dysmetria Consequently, other targets have been sought in cases of MS with tremor.

The zona incerta has dense reciprocal connections with several motor areas in the cortex, subcortical structures and the cerebellum. It has been a lesional target for tremor control in PD in the past. Here we report four cases of MS with complex proximal and distal arm tremor treated by chronic DBS of the contralateral zona incerta. All patients had functional benefit in the use of the affected arm after a minimum follow up of 6 months. The use of field potentials recorded from subcortical nuclei and its correlation with the proximal and distal EMGs from affected limbs in localization of the functional target is described.

\section{METABOLIC ABNORMALITIES IN PARKINSON'S DISEASE: EVIDENCE FOR A NEUROTOXIC CAUSE?}

Newbold SG, Rostami-Hodjegan A, Lennard MS, Tucker GT, Grunewald RA University of Sheffield, UK

Background: The aetiology of Parkinson's disease (PD) has both genetic and environmental contributions. Cytochrome P-450 (CYP) enzymes are involved in chemical detoxification. CYP2D6 poor metaboliser status increases the risk of developing PD. In this study we examined the involvement of CYP1A2 as well as CYP2D6

Methods: The activity of CYP1A2 was determined using caffeine and that of CYP2D6 using debrisoquine as probe drug.

Results: CYP2D6: The patient group $(n=46)$ contained a higher proportion of poor metabolisers than the control group $(n=22)$, $21 \% \vee 5 \%, O R=4$. Enzyme activity was lower in patients than controls (mean log metabolic ratio $1.24 \vee 0.23,95 \% \mathrm{Cl} 0.36-1.67$, $\mathrm{p}<0.005)$.

CYP1A2: The activity of this enzyme was higher in the patient group $(n=53)$ than in the control subjects $(n=32)$ (mean log caffeine:paraxanthine ratio, $20.17 \vee 20.52,95 \% \mathrm{Cl} 0.11-0.59$, $p=0.005$ )

Discussion: The metabolic differences suggest that a putative protoxin could be metabolised to a neurotoxin by CYP1A2, or alternatively detoxified by CYP2D6. The balance of the activities of these two enzymes in an individual could determine the risk of developing PD. As high activity of CYP1A2 is associated with PD, drugs which reduce the activity of this enzyme may have neuroprotective properties. 


\section{FLORID WHITE MATTER ABNORMALITIES ON MRI IN} NEUROACANTHOCYTOSIS

Nicholl DJ, Sutton I, Danek A, Bundey S, Spillane JA, Lawden M Queen Elizabeth Hospital, Birmingham; Leicester Royal Infirmary; Ludwig Maximilians University, Munich

Neuroacanthocytosis is an uncommon neurodegenerative disorder associated with acanthocytosis in the absence of any lipid abnormality. Mutations in at least two genes are responsible for neuroacanthocytosis- the $X K$ gene (in the X-linked McLeod phenotype) and the CHAC gene (on 9q21, in autosomal recessive neuroacanthocytosis). We report widespread white matter abnormalities in two cases of neuroacanthocytosis (one with the McLeod phenotype).

Case 1 was a 61 year old white male who presented with dementia, seizures, facial tics and generalised chorea. Acanthocytes present on repeated blood films with weak Kell antigen. DNA analysis identified a R133X mutation in the XK gene (the McLeod phenotype).

Case 2 was a 32 year old man, born of consanguineous parents, who presented with disinhibition, altered personality and chorea. Acanthocytes present on repeated blood films with negative Kell serology. DNA analysis of the XK and CHAC loci in progress.

$M R I$ in both cases showed widespread areas of increased signal within the white matter of both cerebral hemispheres on T2 weighted imaging.

Previous reports have demonstrated non-specific global atrophy with increased signal on T2 weighted imaging in the caudate and putamen. Our cases suggest that the range of MRI abnormalities in neuroacanthocytosis is much wider than previously thought and can include widespread white matter changes.

\section{A STUDY OF POST-TRAUMATIC CERVICAL DYSTONIA}

O'Riordan S, Hutchinson M St Vincent's University Hospital, Dublin, Ireland

Background and objective: Post-traumatic cervical dystonia (CD-T) may be a distinct syndrome with different characteristics from idiopathic cervical dystonia (CD-I). Our aim is to compare clinical characteristics of these two groups of patients.

Patients and methods: Detailed questionnaires were sent to 121 patients with cervical dystonia attending our neurology clinics.

Results: Response rate was $79 \%$. Twenty five respondents reported neck/shoulder trauma in the year preceding dystonia onset (CD-T); 52 reported no preceding trauma (CD-I), whereas 19 described trauma more than 1 year before dystonia onset (mean interval: 8.2 years, range: $2-26$ years). Female to male ratios in the CD-T and CD-I groups were $3: 2$ and $2: 1$, respectively. Mean age at onset was 42.5 years (range: $18-70$ years) in those with CD-I and 37.5 years (range 18-59 years) for CD-T. Litigation occurred in $49 \%$ of CD-T cases. Sensory tricks were reported less often in those with CD-T $(24 \%$ v $52 \%, p<0.05)$. Mean visual analogue scale scores for pain were significantly higher in the CD-T group $195 \%$ confidence intervals $(95 \% \mathrm{Cl})$ for the difference of the means $[0.3,2.9] ; p<0.05)$ while a poorer response to botulinum toxin was reported $195 \% \mathrm{Cl}$ [20.26,2.46]; $p>0.101$.

Conclusion: Differences between the two groups were observed, suggesting that post-traumatic cervical dystonia may be a distinct clinical entity.

\section{IN VIVO MR STUDIES IN A TRANSGENIC MOUSE MODEL OF HUNTINGTON'S DISEASE}

Page RA, West DA, Cady EB, Thornton JS, Bates GP, Woodman B, Bainbridge A, Priest AN, Ordidge RI, Davie CA Royal Free Hospital; UCL Hospitals; Guy's Hospital, London, UK

The R6/2 mouse is a well documented model of Huntington's disease (HD) and is transgenic for part of the human HD gene. It shows a neurological phenotype progressing to death by around 14 weeks. Histologically it shows brain atrophy and neuronal intranuclear inclusions.

Twenty one R6/2 mice and 17 age matched littermate controls were anaesthetised and studied using localized ' $\mathrm{H}$ MRS and MRI at 7Tesla. ' $\mathrm{H}$ spectra (TR 5s; NA 256) were collected from a $48.5 \mathrm{ml}$ striatal volume ( $T E=135 \mathrm{~ms}$ and $270 \mathrm{~ms})$. Metabolite concentration ratios were obtained using LCModel. Brain water $T_{2}$ was calculated from areas in cortex and basal ganglia.
In R6/2 mice, mean $\mathrm{N}$-acetylaspartate/creatine (NAA/Cr) was reduced $(56.6 \%$ of control mean; $\mathrm{p}<0.005)$, whereas choline/Cr was raised (113.8\%; $\mathrm{p}<0.05)$. Decreased NAA/Cr correlated with age in the R6/2 group (control $r^{2}=0.03 ; \mathrm{R} 6 / 2 r^{2}=0.46$ ). There was no evidence for changes in other metabolites. In both cortex (control=39.6ms, R6/2=38.1; $\mathrm{p}<0.005$ ) and basal ganglia (control=35.7 ms, R6/2=34.0; $p<0.005$ ), brain-water $T_{2}$ was reduced in the transgenic group. There was no significant correlation of $T_{2}$ with age.

The decrease in NAA/Cr parallels the phenotype and underlying neuronal dysfunction. Neuronal loss is not a major feature. Reduced NAA may reflect metabolic neuronal dysfunction, or a reduction in neuronal cell volume, possibly due to dystrophic neurites. Brain-water $\mathrm{T}_{2}$ shortening probably represents dehydration. NAA may provide a surrogate marker for disease progression in the R6/2 strain, facilitating future trials of therapeutic agents.

\section{IDENTIFICATION OF A SYNDROME OF NOCTURNAL RESTLESSNESS IN PARKINSON'S DISEASE USING A NOVEL VISUAL ANALOGUE SLEEP SCALE}

Pal S, DiMarco A, Pezzella FR, Appiah-Kubi L, Trenkwalder C, Ray Chaudhuri K King's College Hospital; GKT School of Medicine; La Sapienza Hospital, Rome; University of Gottingen; University Hospital, Lewisham, UK

Background: It is unclear how frequently nocturnal restlessness occurs in Parkinson's disease (PD) and the extent to which it causes sleep disturbance in PD patients.

Methods: 156 unselected random PD patients $(97$ male, mean age 67.0 (SD 9.7) years, mean disease duration 5.8 years (SD 5.1), mean Hoehn and Yahr 2.7 (SD 0.7) and 46 healthy adults (16 male, mean age 54.2 (SD 12.5)), completed the Parkinson's Disease Sleep Scale (PDSS), a 15 item visual analogue scale addressing nocturnal PD symptoms (0 (symptom severe) to 10 (symptom free)). Scores for items of the PDSS directly pertaining to restlessness (limb restlessness, fidgeting, parasthesias, and painful muscle cramps) were correlated with specific items of the PDSS relating to overall quality of night's sleep, sleep onset, sleep refreshment, and propensity to fall asleep unexpectedly during the day.

Results: The mean total score for four items of the PDSS relating to nocturnal restlessness was significantly lower in PD patients compared to non-parkinsonian healthy adult controls (27.5 (SD 8.1) versus 33.1(SD 9.1), $\mathrm{p}<0.01)$ ) indicating significant nocturnal restlessness. Mean scores for these symptoms also correlated significantly with aspects of sleep disruption and EDS.

Discussion: Nocturnal restlessness is common in PD and causes considerable sleep disruption.

\section{PROSPECTIVE RANDOMISED PLACEBO CONTROLLED CROSSOVER TRIAL OF APOMORPHINE IN PATIENTS WITH LATE STAGE PARKINSON'S DISEASE}

Steiger M, Weiser R, Fox S, Kellett M, Fletcher NA Walton Centre for Neurology and Neurosurgery, Liverpool; Morriston Hospital, Swansea, UK

We assessed the continued effectiveness and safety of apomorphine for "off" periods of immobility in Parkinson's disease in patients with "on-off" motor fluctuations after previous regular usage of the drug for at least 3 months.

The study involved 17 patients with Parkinson's disease in a prospective, randomised, placebo controlled, crossover design. There was a significant peak reduction in UPDRS motor scale at 20 minutes postinjection of 20.0 points, at an average dose of $3.9 \mathrm{mg}$ apomorphine, as compared with a 3.0 point reduction with placebo $(p<0.0001)$. Similar significant reduction in UPDRS was seen at 10 minutes and 60 minutes with apomorphine $(p<0.01$, and 0.005 , respectively). Apomorphine was well tolerated. Mild dyskinesia was frequently associated with the beneficial response to the motor state. All patients had been using apomorphine for greater than 4 months before entry in the study with the average use of apomorphine in patients in the study as 2.14 years.

We conclude that the long term use of apomorphine is not associated with loss of efficacy. 
96 GLOBUS PALLIDUS INTERNUS DEEP BRAIN STIMULATION IN THE TREATMENT OF DYSTONIA

Yianni J, Gregory R, Bain P, Joint C, Parkin S, Aziz T Raddliffe Infirmary, Oxford, UK

Functional neurosurgical intervention is employed for dystonia patients with widely differing disease expressions. The choice of target is based on past evidence of the efficacy of these targets for movement disorders in general. In the current era of functional surgery for movement disorders, deep brain stimulation (DBS) of the globus pallidus internus (GPi) is emerging as the favoured target in patients with dystonia. A retrospective study of 20 consecutive patients with medically intractable dystonia treated with bilateral GPi stimulation is reported. The series comprises 10 patients with generalised dystonia, and six with spasmodic torticollis and four with other types of dystonia. Average follow up was 12 months. Chronic DBS benefited all groups, resulting in clear and stable improvements in their respective neurological rating scales. This study clearly demonstrates that stimulation of the GPi provides amelioration of otherwise untreatable dystonia. Therefore, there is now need for carefully controlled studies to further evaluate the benefit of this treatment.

\section{ACUTE NEUROLOGY IN THE DISTRICT GENERAL HOSPITAL: ARE WE MEETING THE DEMAND, AND CAN WE?}

De Silva R, Senanayake B, Peiris PJP, Findley L, Hawkes CH Essex Centre for Neurology and Neurosurgery, UK

Objectives: To evaluate the neurological input patients with disorders of the nervous system can expect to receive in a "typical" district general hospital (DGH), and to estimate the number of consultant neurologists required to provide an adequate level of care both locally and nationally.

Method: A survey of all patients occupying beds in two acute hospitals serving a population of 450000 , was carried out on a Monday and a Thursday of the same week in August, 2000.

Results: On average, $8.6 \%$ of patients occupied beds primarily with neurological illnesses. Of these, $31 \%$ were under the care of a neurologist. $16.5 \%$ of patients with neurological disorders under the care of non-neurologists were referred for a neurological opinion. $12.5 \%$ of patients with underlying neurological problems, presenting with non-neurological complications, were referred for neurological opinions (none under the care of neurologists).

Conclusions: Less than $50 \%$ of patients presenting primarily with neurological disorders receive specialist (neurological) input. The low rate of referrals of patients with primary neurological problems is probably attributable to the referral habits of (usually) general physicians, which in turn may reflect the shortage of consultant neurologists in the UK. In this DGH the shortfall is in the region of $1.5-7$, and nationally there may be a need for more than 1000 new neurologists.

\section{PATIENT SATISFACTION IN NEUROLOGY OUTPATIENTS: TEACHING HOSPITAL VERSUS DISTRICT GENERAL HOSPITAL}

Gnanapavan S, Cockerell OC Royal London Hospital, London, UK

Patients' assessments of quality of medical care and similar quality assurance activities form an integral part of clinical governance, and a starting point for a more patient centred approach to care.

Using a previously validated Patient Satisfaction Questionnaire (PSQ), we developed a short, self administered questionnaire. The PSQ contained 30 Likert-type items, individual's postvisit satisfaction with access, technical and interpersonal aspects of the neurology outpatient service and satisfaction with the visit overall were ascertained. Responders were asked to select from one of five choices depending on the strength of agreement/disagreement. Questionnaires were administered to 89 new patients presenting for scheduled visits to neurology outpatients at Royal London Hospital and St. Bartholomew's Hospital (teaching hospitals) and Princess Alexandra Hospital and St Margaret's Hospital (DGHs) over a 4-week period.

Patients were in general satisfied with the care provided in the neurology outpatients (81\% satisfied), satisfaction in the DGHs was only marginally higher than in the teaching hospitals compared. Interpersonal skills (conduct and empathy) and technical quality (explanation of problems and examination) of care appeared to be the strongest predictors of overall satisfaction, $r_{s}=0.778$ and 0.735 respectively. However, many patients were dissatisfied with the wait for appointments and clinic waiting times, more so in the teaching hospitals.

In conclusion, PSQs are good way of assessing patient satisfaction in outpatient clinics and our results correlate with known indices of satisfaction.

\section{ABNORMAL BILATERAL CEREBELLAR ACTIVITY IN OVERT MOVEMENT AND MOTOR IMAGERY IN ESSENTIAL TREMOR: A FUNCTIONAL MAGNETIC RESONANCE IMAGING STUDY}

Lindahl AV, Francis S, Morris P, Sawle GV University of Nottingham, UK

It has been postulated that essential tremor (ET) may be caused by a central oscillating motor circuit or by an oscillating circuit involving motor efferent and sensory afferent pathways. Previous functional imaging studies using positron emission tomography (PET) have demonstrated abnormal bilateral cerebellar activation during involuntary tremor in ET, implying that the olivocerebellar pathways have a pivotal role in tremor generation, but not excluding the role of abnormal sensory input. This study used functional magnetic resonance imaging to compare overt and imagined movement in ET.

Eight normal and seven ET patients were studied. All ET patients had postural tremor on arm extension. The functional paradigm incorporated two conditions separated by rest periods: right arm extension and imagined right arm extension.

Both normal subjects and ET patients activated the contralateral motor cortex in response to both overt and imagined right arm extension. The most striking finding was of abnormal bilateral cerebellar activation, not only in response to overt movement, but also in response to imagined movement in the patient group. This supports the role of an oscillating motor circuit in tremor generation in ET and argues against the involvement of abnormal sensory processing in the pathogenesis.

\section{INVESTIGATION OF MITOCHONDRIAL FUNCTION IN HEREDITARY SPASTIC PARAPARESIS}

McDermott CJ, Taylor RW, Hayes C, Johnson M, Bushby KMD, Turnbull DM, Shaw PJ University of Sheffield; University of Newcastle upon Tyne, UK

Introduction: Hereditary spastic paraparesis (HSP) is genetically heterogeneous. Three autosomal genes spastin, paraplegin and atlastin have been identified. Paraplegin is a nuclear encoded mitochondrial metalloprotease. HSP patients with mutations in the paraplegin gene have associated mitochondrial dysfunction. Mitochondrial dysfunction had not previously been considered as a mechanism of neurodegeneration in HSP.

Aims: To investigate the presence of mitochondrial dysfunction in defined genetic populations of HSP

Methods: A needle muscle biopsy was performed on patients previously screened for mutation in both spastin and paraplegin genes. The muscle tissue was analysed histochemically by sequential staining for cytochrome oxidase $\mathrm{c}$ and succinate dehydrogenase. Activities of the respiratory chain complexes (I, II, and IV) in the muscle tissue were assayed spectrophotometrically.

Results: Fourteen patients were recruited in total, five with spastin mutations and nine with both spastin and paraplegin mutations excluded. Twenty aged matched controls were analysed. No statistical difference was identified between the spastin, non-spastin/ paraplegin and control groups by one way ANOVA for either the histochemical or spectrophotometric analysis of muscle tissue.

However in the non-spastin/paraplegin patients we identified subgroups with decreases in mitochondrial complex I activity (three patients) and complex IV activity (one patient). These decreases were greater than twice the SD of the mean complex I or IV activities of the matched control group.

Conclusion: Mutations in the spastin gene are not associated with mitochondrial dysfunction in muscle tissue. The finding of a subgroup in the non-spastin/paraplegin group, with evidence of mitochondrial dysfunction, suggests there are as yet unknown genes, mutation in which cause HSP and mitochondrial dysfunction. 


\section{CHANGES IN OCULOMOTOR STRATEGY DURING VISUAL SEARCH IN PATIENTS WITH FOCAL CORTICAL LESIONS}

Mort DJ, Mannan SK, Hodgson TL, Anderson E, Husain M, Kennard C Imperial College, London, UK

Human beings are highly strategic in how they search, choosing to make a pattern of sequential saccadic eye movements (search path) which complements the perceived visual demands of the search task. We looked in detail at the search paths made during single target visual search by normal adults and patients with frontal and/or parietal cortical lesions.

All subjects first underwent a block of trials where the search target was very difficult to distinguish from distractors ("serial" search), followed immediately by a block where, with the same distractors, the target was easily distinguished ("parallel" search). Though there was a broad range of oculomotor strategy adopted during serial search, all subjects rapidly adjusted this within the parallel search block to account for the decrease in search difficulty, as evidenced by fewer and larger saccades (larger visual attentional field) and a less linearly progressive searchpath.

This strategic adaptation took more trials to emerge in patients with frontal cortical lesions, emphasising a role for the prefrontal cortex in flexible adjustment of oculomotor behaviour. By contrast, a patient with Balint's syndrome from bilateral parieto-occipital damage, who was unable to detect targets of either type easily, searched only with an extremely slowly progressing search path in both serial and parallel search tasks.

\section{ANTIBODIES TO VOLTAGE GATED POTASSIUM CHANNELS IN ENCEPHALITIS}

Palace J, Williams G, Lang B, Vincent A Radcliffe Infirmary; John Radcliffe Hospital, Oxford, UK

Background: Raised levels of antibodies to voltage gated potassium channels (VGKC) have been found in acquired neuromyotonia, Morvan's syndrome (neuromyotonia with CNS involvement), and two patients with limbic syndromes. It is not yet clear how frequently VGKC antibodies are present in patients with CNS disorders, whether neuromyotonia is always present, or whether they signify a good response to immunomodulation.

Methods: Eleven serum samples from patients with unusual adult onset epilepsy syndromes and/or unexplained encephalitic/psychotic symptoms presenting to a general neurology clinic were screened for VGKC antibodies. Results were compared with sera from healthy and disease controls.

Results: Five of 11 sera were positive at levels between 150 and $1050 \mathrm{pM}$ (controls < $100 \mathrm{pM}$ ). The clinical syndromes included: late onset myoclonic epilepsy with cognitive impairment and improvement with plasma exchange; limbic encephalitis with focal siezures; Hashimoto's encephalitis; intractable epilepsy and hypogammaglobulinaemia; and an acute onset of personality change and psychosis. Neuromyotonia was not evident clinically, and was excluded by electrophysiology in one patient.

Discussion: VGKC antibodies appear relatively common in patients with unexplained encephalitic illnesses, but not necessarily neuromyotonia. The lack of such antibodies in other CNS controls and a response to plasma exchange in one of two patients suggests these antibodies may be pathogenic.

\section{DEEP BRAIN STIMULATION OF THE PERIVENTRICULAR GREY IN CHRONIC PAIN: A CLINICAL AND ELECTROPHYSIOLOGICAL STUDY}

Parkin SG, Nandi D, Gregory RP, Smith H, Stein JF, Aziz TZ Radcliffe Infirmary, Oxford, UK

Deep brain stimulation (DBS) for the treatment of chronic central pain has recently been largely supplanted by motor cortex stimulation (MCS). However, MCS is not always effective and so there is still a role for DBS of the central grey matter.

We implanted periventricular grey (PVG) and thalamic electrodes (3389 Medtronic) simultaneously in eight patients with intractable central neuropathic (7 post-stroke, 1 Chiari malformation) pain. Field potentials (FP) were recorded from the sensory thalamus during trial stimulation of the PVG in alert patients. Pain was assessed with a visual analogue scale (McGill-Melzack). Maximum pain relief was obtained with 5-25 Hz stimulation whilst $50-100 \mathrm{~Hz}$ made the pain worse. Interestingly we detected a large amplitude, low frequency thalamic FP at 0.2-0.4 Hz. During 5-25 Hz PVG stimulation the amplitude of this potential was much reduced, but no effect was found at higher $(50-100 \mathrm{~Hz})$ frequencies. Stimulation of the VPL was less effective. Six patients had satisfactory pain relief and were implanted with a stimulator.

Control of central pain with PVG DBS is comparable to that reported for MCS. There is a relationship between thalamic FPs and pain, further study of which may yield insight into the mechanisms of central pain.

\section{SAFETY OF REAL TIME TELEMEDICINE FOR ACUTE NEUROLOGY}

Patterson V, Chua R, Evans H, Russell C Royal Victoria Hospital, Belfast; Tyrone County Hospital, Omagh, Northern Ireland, UK

How neurologists in the UK can help manage patients with neurological symptoms admitted acutely to hospitals is one of the greatest challenges facing UK neurology because neurologist numbers are so low. Real time telemedicine using commercial video conferencing equipment is a possible solution which we have shown is feasible, acceptable, effective and cost effective. Before it will be used widely, however, this unfamiliar method of practice needs to be shown to be safe. We therefore studied 225 consecutive patients seen this way as part of a clinical service between the neurology centre in Belfast and the Tyrone County Hospital in Omagh. The outcome measures that we felt correlated best with safety were deaths, cause of deaths, and changes in diagnosis in a period of 6 months. Information was obtained from a combination of hospital notes, GPs, and patients or their relatives. Four patients were not contactable because they had moved away. Twenty patients died, all of anticipated causes. Diagnosis was changed in 10 patients, and was significant in five. These results are difficult to interpret because of the paucity of information on similar patients seen face to face. Our view is that they show sufficient safety to allow us to increase the use of this technique.

\section{THE EXCITABILITY OF MUSCLE: IN THE FOOTSTEPS OF FRANCIS GLISSON (1597-1677)}

Pearn J, Gardner-Thorpe C Children's Hospital, Brisbane, Australia; Exeter Neurosciences and Peninsula Medical School, UK

The intrinsic properties of cellular irritability are central to neuroscience. Irritability and its precursor, excitability, are two specific properties of mammalian nerve and muscle cells. The pioneer of the concept of intrinsic tissue irritability was a physician better known for the capsule of the liver that was named after him, Francis Glisson (1597-1677).

Glisson was born at Rampisham in Dorset, studied in Oxford, and then in 1636 became Regius Professor of Physic at Cambridge. $\mathrm{He}$ worked also in London around the time of the Great Plague (1665) and the Great Fire of London (1666). In 1667, 1668, and 1669 he held the post of President of the Royal College of Physicians. His thesis on muscle irritability, published in 1677, was a datum point on the path of knowledge that led to the modern disciplines of neuroanatomy, neurophysiology, and clinical neuromuscular disease.

In muscle cells irritability and excitability lead to movement at cellular, tissue and organ level. The intervening steps of membrane depolarisation, intracellular ion flux and transformational change in protein molecule configuration are and will remain the focus of continuing research in these disciplines.

In 1752, some 80 years after Glisson's hypothesis, Albrecht von Haller (1708-77) showed by experiment that muscle possesses intrinsic irritability and contractility. Glisson's Thesis and Haller's findings received their final confirmation in 1850 when Claude Bernard's (1813-78) studies in experimental pharmacology demonstrated that muscle was a functional unit, independent of (though controllable by) electrical stimuli mediated via nerve pathways.

This geographic and scholastic walk in the footsteps of Francis Glisson provides a new perspective of his work. 


\section{A COMPARISON OF BILATERAL SIMULTANEOUS AND BILATERAL SEQUENTIAL OPTIC NEURITIS USING VISUAL PSYCHOPHYSICS}

Pye EM, Weatherby SJ, Kesson O, Foster DH, Hawkins CP Keele MS Research Group, Stoke-on-Trent, UK

Bilateral optic neuritis (BON) affects the eyes simultaneously (BsimON) or sequentially (BseqON). Pathogenesis may differ. Visual psychophysics provides an established measure of sensory deficit which may be interpreted in terms of large and small fibre pathways.

Visual psychophysical testing was performed on four patients (seven eyes) with BsimON, ie, both eyes affected within 1 week, four patients (eight eyes) with BseqON, ie, second eye affected $=3$ months after the first (range 3-72), and five controls (10 eyes). Mean time from optic neuritis onset to psychophysical testing was 41 months for BsimON and 31 months for BseqON. Contrast thresholds were measured at three spatial frequencies $(0.25,1.0$, $4.0 \mathrm{cyc} / \mathrm{deg}$ ) with counterphase and in phase red and green gratings.

Visual acuity recovered to $=6 / 9$ in $5 / 7$ BsimON eyes and in 7/8 BseqON eyes but all had thresholds higher than controls at all spatial frequencies. Mean thresholds were seven times greater than controls in BsimON eyes $(p<0.0001)$, although not significantly different from controls in BseqON eyes $(p=0.4)$. BsimON eyes appeared to have a preferential parvocellular deficit at $0.25 \mathrm{cyc} / \mathrm{deg}$ $(p=0.05)$.

Despite good recovery of visual acuity in most eyes, visual psychophysical thresholds were much higher in BsimON than BseqON. The small fibre parvocellular pathway seemed preferentially affected.

\section{SIMULTANEOUS ENCEPHALITIS AND ACUTE RETINAL NECROSIS SECONDARY TO VARICELLA ZOSTER AND HERPES SIMPLEX VIRUS}

Samuel M, Davies N, Pavesio CE, Graham E, Plant GT National Hospital for Neurology and Neurosurgery; Moorfields Eye Hospital; St Thomas' Hospital, London, UK

Introduction: Acute retinal necrosis (ARN) in immunocompetent patients is associated with reactivation of Herpes viruses. Previously reported cases suggest an association with encephalitis, with the encephalitis occurring years or decades before ARN. We describe two cases of simultaneous occurrence of encephalitis with ARN.

Methods: A 59 year old woman developed dysphasia and confusion acutely, without fever or alteration of consciousness. She had been in previously good health, but was a smoker. Brain MRI showed left temporal lobe signal changes, initially interpreted as an infarct. Her condition remained stable but 3 weeks later, she developed bilateral visual failure. Ocular examination was suggestive of ARN. Repeat brain MRI showed bilateral temporal lobe signal abnormalities, suggestive of viral encephalitis. CSF examination was normal, including PCR for viral nucleic acids, but local synthesis of oligoclonal lgG was detected. Immunoblotting of the lgG bands confirmed specificity against VZV and HSV antigen. We have found one other case from our records who presented with encephalitis, followed shortly by ARN.

Conclusion: Cerebral symptoms followed shortly by ARN should raise the possibility of herpetic encephalitis, even in the absence of a positive CSF PCR test for these viruses.

\section{MITOCHONDRIAL DISEASE AND SYMPTOM PROGRESSION: A COMPLEX MANAGEMENT ISSUE}

Schaefer AM, Taylor RW, Macfarland RM, Chinnery PF, Turnbull DM University of Newcastle-upon-Tyne, UK

Background: Mitochondrial DNA defects are an important cause of neurological disease. Their clinical course is extremely variable and this creates many difficulties in follow-up and early detection of new and potentially treatable complications. We aim to further define the clinical course associated with the MELAS (mitochondrial encephalomyopathy, lactic acidosis and stroke-like episodes) a3243g mutation.
Methods: We took 43 patients with a known genetic diagnosis of MELAS. Clinical data was obtained prospectively, documenting the development of symptoms. In all we were able to obtain over 200 years of prospective data.

Results: We document both a definite disease progression and the development of potentially treatable complications such as diabetes, hypertension and left ventricular hypertrophy. Certain age groups show an increased incidence of these complications while males have earlier disease progression.

Discussion: Many of our patients developed both neurological and non-neurological complications, many of which are amenable to early treatment. Age groups showing a higher incidence of these complications may warrant more frequent screening. Interestingly, analysis suggests accelerated progression in male subjects suggesting that these patients may also gain an increased benefit from regular screening measures.

\section{A NOVEL FORM OF LOCALISED AMYLOID DEPOSTION CAUSING A PROGRESSIVE MYELOPATHY ASSOCIATED WITH CORNEAL LATTICE DYSTROPHY}

Talbot K, Bron A, Akhtar S, Donaghy M Radcliffe Infirmary, Oxford; Cardiff University, Cardiff, UK

Background: A family presented with a novel phenotype of wasting of the small muscles of the hands associated with painless visual loss, inherited as an autosomal dominant trait. The age of onset was variable (second to fifth decade). At least one family member has gone on to develop a moderately disabling myelopathy and the corneal disease ultimately requires grafting.

Methods: A clinical and genetic study was performed to investigate the underlying basis of the association between the neurological and ophthalmological features.

Results: All known genes associated with corneal lattice dystrophy were screened for mutations but this proved negative. MRI scanning showed focal high signal in the cervical spinal cord but no compression. Electron microscopy of the cornea has demonstrated focal deposition of insoluble amyloid protein. Nerve conduction and EMG studies indicated that the disease process predominantly caused denervation of affected muscles with normal sensory and motor conduction.

Discussion: This is the first report of a novel form of neurological disease associated with corneal lattice dystrophy due to amyloid deposition. Further work is underway to characterise the nature of the amyloid protein and to identify the genetic basis of this disorder.

\section{AXONAL DEPOLARISATION IN CHRONIC RENAL FAILURE}

Walters RJL, Kiernan MC, Taube D, Andersen KV, Murray NMF, Bostock $\mathrm{H}$ The National Hospital for Neurology and Neurosurgery, London, UK

Introduction: Axonal sensorimotor neuropathy is common in patients starting haemodialysis for chronic renal failure (CRF). The pathophysiology of uraemic neuropathy remains uncharacterised but one potential mechanism is chronic axon depolarisation secondary to the effects of "middle weight" or other neurotoxins. This can be investigated by recently developed neurophysiological techniques that examine axonal excitability and are thus particularly sensitive to resting nerve membrane potential.

Method: Excitability studies were performed on nine patients before their regular haemodialysis, stimulating the median nerve and recording from the abductor pollicis brevis. Six of the nine patients had further recordings during and after dialysis.

Results: The predialysis results revealed significant depolarisation, when compared with normal subjects, reflected by abnormally increased refractory period, reduced superexcitability and reduced threshold electrotonus to depolarising and hyperpolarising currents (all $p<0.0005$ ). These abnormalities were substantially reversed by dialysis. CRF patients had abnormalities in a number of biochemical parameters but only potassium concentration correlated with axon depolarisation.

Conclusion: Axons in CRF patients are depolarised due to hyperkalaemia. Since axons require adequate membrane polarisation to maintain normal biochemical homeostasis, this chronic depolaris. 
Clinico-pathological conference for Spring ABN meeting, Oxford

Case presented by: Dr K Talbot

Discussed by: Dr N Fletcher

Pathology demonstrated by: Professor M Esiri

\section{A YOUNG WOMAN WITH PROGRESSIVE RIGIDITY}

Case history: The patient, an office administrator for a spray painting firm, was referred as an urgent case by her GP, two days after her 26th birthday. His letter documented a 2-3 month history of positional vertigo, lethargy and a feeling that she was "slowing up". This was associated with slurring of speech and mild clumsiness. Her husband stated that she has been talking in her sleep for the few weeks prior to admission. She was admitted to the neurology ward a few days later because she had been vomiting and an expanding intracranial lesion was suspected. The history was as above but the vertigo is described as occurring on getting up from sitting to standing and to last for 10-15 seconds with the room spinning round. She was noted to be finishing a course of chloroquine, having just returned from a 2 week holiday in Sri Lanka. She and her husband felt that the onset of her symptoms predated her trip. There was no PMH. Her mother died of ovarian carcinoma at 57, her father was alive and well; there were no sibs. Bedside testing of higher function was normal. Physical examination revealed moderate axial rigidity and a blank face. Vertical gaze was slow and incomplete. The tone in the limbs was normal. There was mild finger nose incoordination, but no weakness. The reflexes were felt to be generally brisk with three beats of clonus at both knees and ankles. The plantar responses were flexor. She was placed on thiamine $100 \mathrm{mg} /$ day. The following investigations were normal: FBC and blood film, U and Es, LFTs, Lactate/pyruvate, full autoantibody screen including ANCA, copper studies, slit lamp examination, MRI brain, bone marrow biopsy, muscle biopsy, anti-neuronal antibodies, ultrasound of the pelvis and abdomen, mammography, CSF and serum viral serology, upper Gl endoscopy and biopsy. She was discharged 2 weeks after admission.

She was readmitted 2 weeks later for further investigations. She was more lethargic and tearful. The physical signs were unchanged except for increased tone in the upper limbs and increased dysarthria. Over the following months she developed progressive stiffness, dysarthria and motor slowing, excessive sweating and disturbed temperature regulation and slept 20 hours a day. After several months she developed a positive glabellar tap, intermittent blepharospasm, and an increasing impairment of vertical gaze. A trial of sinemet produced no clinical benefit. A right frontal biopsy of the brain was performed. She continued to decline and died 10 months after the initial referral.

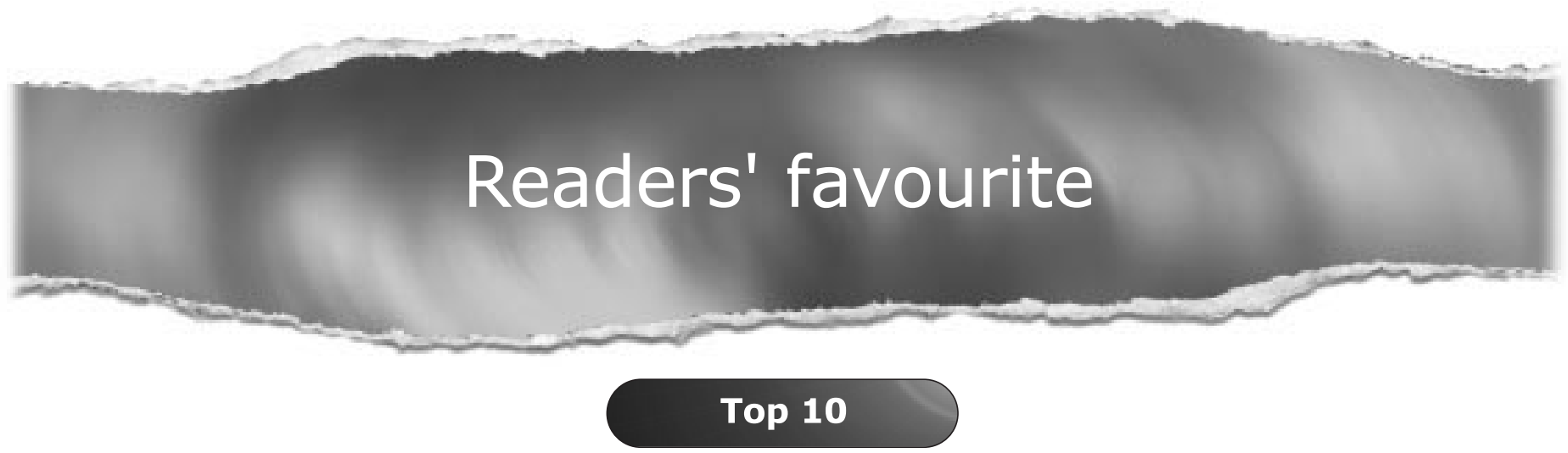

Click on the "Top 10" button on the homepage to see which are the best read articles each month

www.jnnp.com 